\title{
Spatially Resolved Analysis of Neutral Winds, Stars, and Ionized Gas Kinematics with MEGARA/GTC: New Insights on the Nearby Galaxy UGC 10205
}

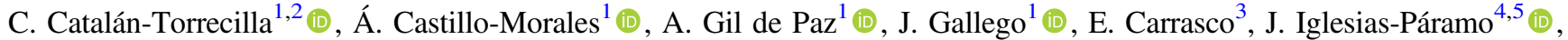 \\ R. Cedazo ${ }^{6}$, M. Chamorro-Cazorla ${ }^{1}$, S. Pascual ${ }^{1}$ (i), M. L. García-Vargas ${ }^{7}$, N. Cardiel ${ }^{1}$ (1) , P. Gómez-Alvarez ${ }^{7}$, A. Pérez-Calpena ${ }^{7}$, \\ I. Martínez-Delgado ${ }^{7}$, B. T. Dullo ${ }^{1}$ (1), P. Coelho ${ }^{8}$ (1) , G. Bruzual $^{9}$ (i) , and S. Charlot ${ }^{10}$ (1) \\ ${ }^{1}$ Departamento de Física de la Tierra y Astrofísica, Instituto de Física de Partículas y del Cosmos IPARCOS, Universidad Complutense de Madrid, E-28040 Madrid, \\ Spain; ccatalan@ucm.es \\ ${ }^{2}$ Centro de Astrobiología (CAB, CSIC-INTA), Carretera de Ajalvir km 4, E-28850 Torrejón de Ardoz, Madrid, Spain \\ ${ }^{3}$ Instituto Nacional de Astrofísica, Óptica y Electrónica, Luis Enrique Erro No. 1, C.P. 72840, Tonantzintla, Puebla, Mexico \\ ${ }_{5}^{4}$ Instituto de Astrofísica de Andalucía-CSIC, Glorieta de la Astronomía s/n, E-18008, Granada, Spain \\ ${ }^{5}$ Estación Experimental de Zonas Áridas-CSIC, Ctra. de Sacramento s/n, E-04120 Almería, Spain \\ ${ }^{6}$ Universidad Politécnica de Madrid, Madrid, Spain \\ ${ }^{7}$ FRACTAL S.L.N.E. C/Tulipán 2, p13, 1A. E-28231, Las Rozas de Madrid, Spain \\ ${ }^{8}$ Universidade de São Paulo, Instituto de Astronomia, Geofísica e Ciências Atmosféricas, Rua do Matão 1226, 05508-090, São Paulo, Brazil \\ 9 Instituto de Radioastronomía y Astrofísica, UNAM, Campus Morelia, Michoacan, México, C.P. 58089, México \\ ${ }^{10}$ Sorbonne Université, CNRS, UMR7095, Institut d'Astrophysique de Paris, F-75014, Paris, France \\ Received 2019 August 8; revised 2019 December 9; accepted 2019 December 17; published 2020 February 6
}

\begin{abstract}
We present a comprehensive analysis of the multiphase structure of the interstellar medium (ISM) and the stellar kinematics in the edge-on nearby galaxy UGC 10205 using integral field spectroscopy (IFS) data taken with MultiEspectrógrafo en GTC de Alta Resolución para Astronomía (MEGARA) at the GTC. We explore both the neutral and the ionized gas phases using the interstellar Na I D doublet absorption (LR $-V$ setup, $R \sim 6000$ ) and the $\mathrm{H} \alpha$ emission line (HR $-R$ setup, $R \sim 18000$ ), respectively. The high-resolution data show the complexity of the $\mathrm{H} \alpha$ emission-line profile revealing the detection of up to three kinematically distinct gaseous components. Despite of this fact, a thin-disk model is able to reproduce the bulk of the ionized gas motions in the central regions of UGC 10205. The use of asymmetric drift corrections is needed to reconciliate the ionized and the stellar velocity rotation curves. We also report the detection of outflowing neutral gas material blueshifted by $\sim 87 \mathrm{~km} \mathrm{~s}^{-1}$. The main physical properties that describe the observed outflow are a total mass $M_{\text {out }}=(4.55 \pm 0.06) \times 10^{7} M_{\odot}$ and a coldgas mass outflow rate $\dot{M}_{\text {out }}=0.78 \pm 0.03 M_{\odot} \mathrm{yr}^{-1}$. This work points out the necessity of exploiting highresolution IFS data to understand the multiphase components of the ISM and the multiple kinematical components in the central regions of nearby galaxies.
\end{abstract}

Unified Astronomy Thesaurus concepts: Extragalactic astronomy (506); Galaxies (573); Stellar kinematics (1608); Spectroscopy (1558); Galaxy winds (626)

\section{Introduction}

As it is a fundamental physical process for galaxy evolution models, the ubiquity of galaxy-scale outflows across all cosmic epochs has been probed through vast observational evidence (see reviews by Veilleux et al. 2005; Rupke 2018). Stellar winds, supernovae, or active galactic nuclei (AGNs) have been postulated as the potential mechanisms to drive these phenomena to which we will broadly refer as Galactic winds.

Galactic winds are candidates to contribute to the metal enrichment of the intergalactic medium (Nath \& Trentham 1997; Dalcanton 2007). The gas entrained in the wind may escape the halo potential (Heckman et al. 2000), or it could fall back down onto the galaxy in a process referred to as galactic fountain (Shapiro \& Field 1976). Galactic winds are essential for understanding the feedback mechanisms and their impact on the host galaxy. While they might produce a positive effect in which the compression of the interstellar and circumgalactic medium can potentially boost the star formation (Nayakshin \& Zubovas 2012; Zubovas et al. 2013), they can also be considered as a plausible mechanism to suppress star formation within galaxies (Granato et al. 2004; King \& Pounds 2015).

Given their intrinsic multiphase nature, different tracers (Xrays, optical nebular emission lines, ISM absorption lines, CO transitions) ought to be used to characterize their different phases (hot, warm, cold, and molecular). While most relevant studies have traditionally focused on the study of Galactic winds on extreme objects, such as luminous and ultraluminous infrared galaxies (LIRGs and ULIRGs), mergers, and strong starbursts (Martin 2005; Rupke et al. 2005; Rupke \& Veilleux 2005; Arribas et al. 2014; Cazzoli et al. 2014, 2016), the presence of relatively strong winds in less active galaxies has remained mostly unexplored (Castillo-Morales et al. 2007; Jiménez-Vicente et al. 2007; Chen et al. 2010; Roberts-Borsani \& Saintonge 2019). Within this scenario, the use of new instrumental capabilities to explore the existence, frequency, and strength of winds in relatively quiet galaxies seems to be the necessary next step.

The advent of the Multi-Espectrógrafo en GTC de Alta Resolución para Astronomía (MEGARA; A. Gil de Paz et al. 2020, in preparation) will improve the detectability of Galactic winds, helping to separate them from their hosts with exquisite detail. These high-quality Integral Field Spectroscopy (IFS) data are fundamental to kinematically discriminate both the ionized medium and the cold interstellar gas phase of winds and their geometry. That is why, the characterization of Galactic winds in the central regions of nearby galaxies is one of the main scientific objectives of the MEGARA instrument (Carrasco et al. 2018). 
In this context, UGC 10205 was selected as part of the nighttime commissioning observations due to the presence of residual Na I D interstellar absorption previously identified in the CALIFA survey (Sánchez et al. 2012). In the optical range, neutral gas motions are commonly studied using this interstellar medium (ISM) $\mathrm{NaI} \mathrm{D}$ absorption-line doublet at 5889.95 and $5895.92 \AA$. The lower value of the ionization potential for the $\mathrm{NaI} \mathrm{D}(5.14 \mathrm{eV})$ in comparison with the one for the hydrogen makes it an ideal candidate to trace the cool $\left(T<10^{4} \mathrm{~K}\right)$ neutral gas.

In addition, by examining the multiple components of the ISM in galaxies, such as the case of UGC 10205, where mergers might have played an important role (Reshetnikov \& Evstigneeva 1999), one could uncover important clues on the impact of these events on their evolution. Past long-slit spectroscopy studies limited to a few directions only (see Vega Beltran et al. 1997) have revealed the complexity of this object. The use of high-resolution IFS data is essential to provide a comprehensive view on the dynamics of this galaxy. In this work, we perform a reconstruction of the stellar kinematics and gas morphology in the central regions of this nearby galaxy. Thus, UGC 10205 is intended to provide an exploratory study to define the final selection for the optimal exploitation of the MEGARA capabilities both in terms of the stellar and gas kinematics.

The structure of this paper is as follows: Section 2 describes the general properties of the UGC 10205 galaxy, and Section 3 presents MEGARA observations and data reduction. Section 4 outlines the various steps in our analysis, while Section 5 describes the main results of this study including details on the kinematics of the ionized gas and the stellar component and the properties of the cold gas. Finally, we discuss the proposed scenario for this object in Section 6, and we summarize our conclusions in Section 7. Unless otherwise stated, throughout this paper, we adopt a flat $\Lambda$ CDM cosmology with $H_{0}=$ $70 \mathrm{~km} \mathrm{~s}^{-1} \mathrm{Mpc}^{-1}, \Omega_{m}=0.3$ and $\Omega_{\Lambda}=0.7$.

\section{General Properties of UGC 10205}

UGC 10205 is an edge-on disk galaxy classified as an Sa/S0 galaxy by Nilson (1973), Tarenghi et al. (1994), and Nair \& Abraham (2010), respectively, with an inclination of $i=84^{\circ}$ (Rubin et al. 1985). A Sloan Digital Sky Survey (SDSS) composite image for this galaxy appears in Figure 1 showing the presence of dust lanes roughly parallel to the major axis of the galaxy. A shell structure in the outer regions of this galaxy was also discovered by the early studies of Rubin (1987).

The main properties of UGC 10205 are summarized in Table 1 and briefly described below. A systemic heliocentric radial velocity of $6556 \mathrm{~km}^{-1}$ was calculated in Theureau et al. (1998) using measurements of the $21 \mathrm{~cm}$ neutral hydrogen line carried out with the meridian transit Nançay radiotelescope. Walcher et al. (2014) used Bruzual \& Charlot (2003) stellar population models with a Chabrier (2003) stellar IMF to construct a UV to NIR spectral energy distribution to estimate a total stellar mass of $\log \left(M_{\star} / M_{\odot}\right)=10.997 \pm 0.111$. A set of panchromatic luminosity measurements can be also found in the literature for this galaxy. In particular, a far-infrared luminosity of $\log \left(L_{\mathrm{FIR}} / L_{\odot}\right)=10.10$ was reported by Vlahakis et al. (2005) using the Submillimetre Common-User Bolometer Array (SCUBA) data and a $\log \left(L_{\mathrm{TIR}} / L_{\odot}\right)=10.35$ by Willmer et al. (2009) combining Spitzer Space Telescope observations with SCUBA $850 \mu \mathrm{m}$ data. UGC 10205 was observed as part
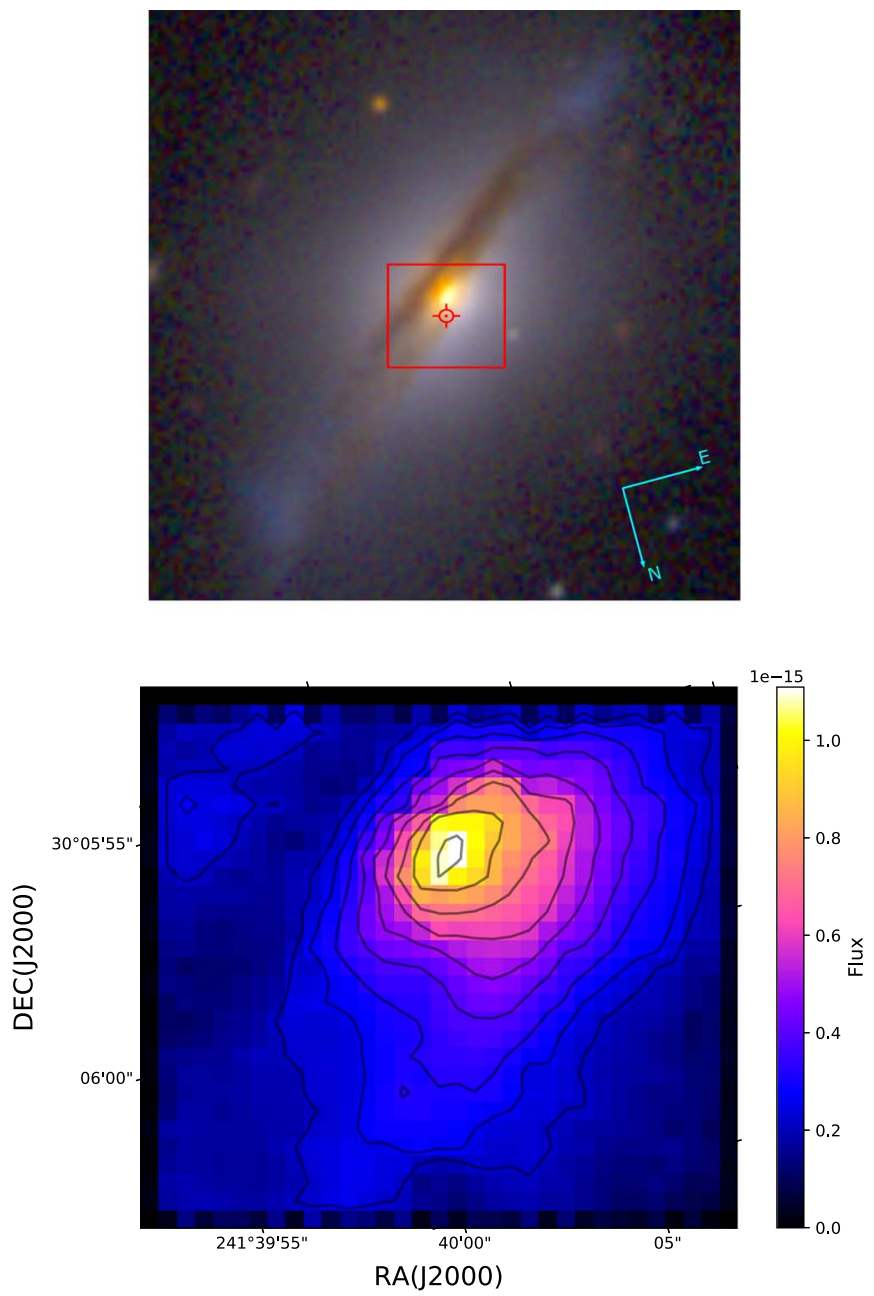

Figure 1. Top panel: SDSS composite image of UGC 10205. Overlaid on the image is the footprint of the MEGARA IFU field of view (red rectangle) covering a region of $5.1 \times 5.7 \mathrm{kpc}^{2}$. The orientation is indicated in the bottomright corner. Bottom panel: distribution of the continuum emission of UGC 10205 derived from the MEGARA IFS data cube in the spectral range $5600-5700 \AA$. The flux units are erg s $\mathrm{cm}^{-1} \mathrm{~cm}^{-2}$ pixel $^{-2} \AA^{-1}$. Pixel size is 0 "! 4 in this case. The area of these synthetic spaxels was chosen as the best compromise between the seeing of the observing nights and the routine used to create the data cubes (see more details in Section 4.2).

Table 1

Global Properties of UGC 10205

\begin{tabular}{lcc}
\hline \hline Property & UGC 10205 & References \\
\hline Morphology & $\mathrm{Sa} / \mathrm{S} 0$ & $(1)(2) /(3)$ \\
$z$ & 0.02187 & $(4)$ \\
R.A.(J2000) & $16^{\mathrm{h}} 06^{\mathrm{m}} 40^{\mathrm{s}} .180$ & $(4)$ \\
Decl.(J2000) & $+30^{\mathrm{d}} 05^{\mathrm{m}} 56^{\mathrm{s}} .70$ & $(4)$ \\
PA (deg) & 135 & $(4)$ \\
$r-$ SDSS diameter (") & 105.3 & $(4)$ \\
Inclination $($ deg $)$ & 84 & $(5)$ \\
$\log \left(M_{\star} / M_{\odot}\right)$ & 10.997 & $(6)$ \\
$\log \left(L_{\mathrm{TIR}} / L_{\odot}\right)$ & 10.35 & $(7)$ \\
$L_{\mathrm{H} \alpha}\left(\mathrm{erg} \mathrm{s}^{-1}\right)$ & $10.35 \times 10^{40}$ & $(8)$ \\
$L_{\mathrm{FUV}}\left(\mathrm{erg} \mathrm{s}^{-1}\right)$ & $2.71 \times 10^{42}$ & $(8)$ \\
\hline
\end{tabular}

Note. (1): Nilson (1973), (2): Tarenghi et al. (1994), (3): Nair \& Abraham (2010), (4): NASA/IPAC Extra-galactic Database, (5): Rubin et al. (1985), (6): Walcher et al. (2014), (7): Willmer et al. (2009), (8): Catalán-Torrecilla et al. (2015). 
of the CALIFA survey (Sánchez et al. 2012). In this context, Catalán-Torrecilla et al. (2015) derived a global $L_{\mathrm{H} \alpha, \mathrm{obs}}=$ $(10.35 \pm 0.57) \times 10^{40} \mathrm{erg} \mathrm{s}^{-1}$ and a $L_{\mathrm{FUV}, \mathrm{obs}}=(2.71 \pm$ $0.67) \times 10^{42} \mathrm{erg} \mathrm{s}^{-1}$ from the Galaxy Evolution Explorer (GALEX, Martin 2005).

Questions arise about the complexity of this object as has been already manifested by several authors. The numerical simulations run by Reshetnikov \& Evstigneeva (1999) show the capture and tidal disruption of a companion (a small-type $\mathrm{E} / \mathrm{S} 0$ ). Other authors have considered this galaxy as a possible polar-ring candidate (Whitmore et al. 1990; van Driel et al. 2000). A profound study done by Vega Beltran et al. (1997) using long-slit spectroscopy data revealed the presence of up to three kinematically distinct gaseous components. The use of IFS data will help to discriminate the spatial distribution of these components and to overcome limitations associated with the use of one-dimensional velocity curves. At a distance of $150 \mathrm{Mpc}\left(H_{0}=75.2 \pm 3.3 \mathrm{~km} \mathrm{~s}^{-1} \mathrm{Mpc}^{-1}\right.$, Sorce et al. 2014), the central $5.7 \times 5.1 \mathrm{kpc}^{2}$ of UGC 10205 can be mapped with MEGARA. These observations have the potential to shed some light on the multiphase components of the ISM to understand the current state and future evolution of this galaxy.

\section{Observations: MEGARA IFS Data}

The spectrophotometric observations of UGC 10205 were carried out with MEGARA (A. Gil de Paz et al. 2020, in preparation) on the $10.4 \mathrm{~m}$ GTC telescope at La Palma Observatory. MEGARA is composed of an Integral Field Unit (IFU) and a Multi-Object Spectroscopy mode. For the purpose of this work, we used only IFU data taken on 2017 June 30 and July 1 as part of the science verification of its commissioning phase. The IFU unit provides a field of view (FoV) of 12 !" $5 \times 11$ !" 3 using 567 hexagonal spaxels of 0 ". 62 in size, which makes it ideal for studying the central regions of nearby galaxies. Due to reasons explained later in Section 4.2, the analysis of the data will be performed using squared spaxels. A recent example showing the potential of MEGARA data can be found in Dullo et al. (2019).

The values of the seeing during the observations ranged between approximately 0 ".93 and 1 "' 11 as recorded by the differential image motion monitor close to the Telescopio Nazionale Galileo. Four $1200 \mathrm{~s}$ science exposures were combined for the $\mathrm{LR}-V$ data set while in the $\mathrm{HR}-R$ setup, a total integration time of $2200 \mathrm{~s}$ were made in two exposures. These data provide a unique view into the cold and warm phases of the ISM. In particular, the LR $-V$ grating is optimal to analyze the ISM Na I $\lambda \lambda 5890,5896$ doublet (Na I D) as it yields a wavelength coverage from $5143 \AA$ to $6164 \AA$ with a spectral resolution of FWHM $=0.937 \AA$ at central wavelength $\lambda_{c}=5695 \AA$. The $\mathrm{HR}-R$ grating provides a wavelength coverage from $6445 \AA$ to $6837 \AA$ (FWHM $=0.355 \AA$ at $\lambda_{c}=6646 \AA$ ) that allows us to characterize the ionized gas by means of the $\mathrm{H} \alpha$ emission line. The reciprocal dispersion is $0.27 \AA \mathrm{pix}^{-1}$ and $0.097 \AA \mathrm{pix}^{-1}$, respectively for $\mathrm{LR}-V$ and $\mathrm{HR}-R$.

Additional images were acquired to help in the data reduction process. In particular, the spectrophotometric standard star BD+33 2642 was observed and used to create a response function to calibrate the absolute flux scale. Arc calibration lamp frames and twilight sky flat-field/lamp flatfield were obtained at the beginning and at the end of each night, respectively. Finally, the reduction of the raw data comprises the standard procedures of bias subtraction, cosmicray removal, flat-fielding, tracing and extraction of the spectra, arc calibration solutions, sky-subtraction, and flux calibration. It was performed applying the standard MEGARA pipeline (Cardiel \& Pascual 2018; Pascual et al. 2018).

\section{Analysis}

\subsection{Galaxy Continuum Subtraction and Interstellar Na I D Detection}

In this section, we describe the main characteristics of the process followed to model the galaxy continuum for the case of the $\mathrm{LR}-V$ grating. As commented previously, the $\mathrm{LR}-V$ covers the range from 5143 to $6164 \AA$ with a spectral resolution of FWHM $=0.937 \AA$ at $\lambda_{c}=5695 \AA$.

First, a Voronoi binning algorithm (Cappellari \& Copin $2003)$ is applied to increase the signal-to-noise ratio $(\mathrm{S} / \mathrm{N})$ of the spectra in the individual spaxels, reaching an $\mathrm{S} / \mathrm{N} \sim$ 20 over the continuum. This process allows for a more homogeneous distribution of the $\mathrm{S} / \mathrm{N}$ within the data cube. Then, we model the galaxy continuum in the spatially binned MEGARA data cube using a Penalized Pixel-Fitting method (pPXF; Cappellari \& Emsellem 2004; Cappellari 2017). The aim of pPXF is to build the stellar template that will best reproduce the observed spectrum in each Voronoi cell. For that purpose, we used the latest theoretical stellar library by Coelho (2014) and P. Coelho et al. (2020, in preparation), which represents an expansion of the previous stellar population modeling in Coelho et al. $(2005,2007)$ and Walcher et al. (2009). The resolution of these models, $R=20000$, is optimal for all of the MEGARA setups and especially for the highestresolution VPHs $(R \approx 20000)$. We selected a total of 660 models with different ages ranging from $30 \mathrm{Myr}$ to $14 \mathrm{Gyr}$ and three different metallicities $([\mathrm{Fe} / \mathrm{H}]=-1.0,0.0,0.2)$ at both scaled-solar and $\alpha$-enhanced mixtures. The stellar continuum was fitted over the spectral range (5150-6160) $\AA$, which contains multiple stellar absorption lines. We carefully masked the ISM features, as they are the result of both the stellar and ISM contribution. Also, the He I emission line at $5875.67 \AA$ was masked due to its proximity to the Na I D line. Finally, the best stellar model was subtracted from the observed spectra to detect the presence of $\mathrm{Na} I \mathrm{D}$ interstellar absorption. To assess the robustness of the pPXF fits within the region measured and its impact on the results, we have applied a standard bootstrap analysis (see Wu 1986) using 100 simulations to each of the Voronoi spectra. First, a redistribution of the best-fit model residuals is done. Then, we fitted the resampled spectrum using pPXF. For the two first LOSVD moments ( $V$ and $\sigma$ ), we obtained dispersions of up to 9 and $10 \mathrm{~km} \mathrm{~s}^{-1}$ after running the 100 repetitions, respectively. We also found mean differences of only $7 \mathrm{~km} \mathrm{~s}^{-1}\left(5 \mathrm{~km} \mathrm{~s}^{-1}\right)$ between the original velocities (stellar velocity dispersions) values and the ones obtained after applying the bootstrapping technique.

Figure 2 shows a representative fit for one of the Voronoi cells. The position of this particular Voronoi cell within the galaxy can be seen in the inset of the figure. The black line is the observed spectrum, while the red line is the fit for the stellar component. The vertical blue shaded regions show the ISM features and sky-lines that have been masked in the fitting process. The residuals of the fitting appear in green at the bottom. There is a clear detection of ISM Na I D absorption in this spectrum. 


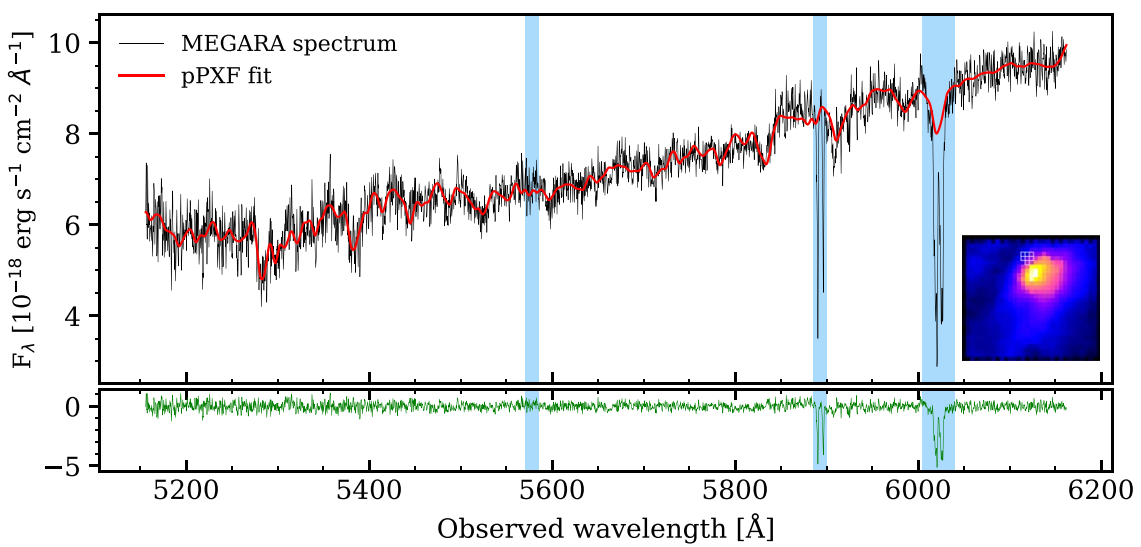

Figure 2. Spectrum for the LR- $V$ MEGARA data cube is shown in black while its best fit from pPXF appears in red. The green spectrum at the bottom represents the fit residuals (note the different vertical scale). The blue shaded areas correspond to the masked regions (ISM features and sky line residuals). The inset shows the position of the Voronoi cell corresponding to this spectrum on the same continuum image of the galaxy as in Figure 1.

The analysis described in this section allows us (i) to derive fundamental parameters of the galaxy kinematics such as the first four order moments of the line-of-sight velocity distribution $\left(V, \sigma, h_{3}\right.$, and $\left.h_{4}\right)$ for each Voronoi cell of the MEGARA data cube (Section 5.2), and (ii) to explore the presence of interstellar Na I D absorption in UGC 10205 and its connection with neutral gas outflows (Section 5.5).

\subsection{Recovering the $H \alpha$ Emission}

In the following paragraphs, we describe the analysis performed to obtain a reliable measure of the $\mathrm{H} \alpha$ emission flux for the case of the HR $-R$ grating. This setup is characterized by wavelength coverage from $6445 \AA$ to $6837 \AA$ and an FWHM = $0.355 \AA$ at $\lambda_{c}=6646 \AA$. The $\mathrm{HR}-R$ observations were taken on different nights, 2017 June 30 and July 1, and the pointing was slightly offset between them (we remind the reader that the data were taken as part of the Commissioning Time). Thus, the first step was to combine both observations to create a new data cube that takes into account the relative offsets between them.

The second step was to remove the stellar continuum of the galaxy. However, due to the lack of absorption features in the wavelength range covered by the HR $-R$ setup, the use of codes such as PPXF is not recommended. For that reason, the method applied for the HR $-R$ case slightly differs from the one used in the LR $-V$ grating (Section 4.1). The approach followed here to estimate the $\mathrm{H} \alpha$ absorption-corrected emission flux is to derive (i) the equivalent width of the stellar $\mathrm{H} \alpha$ absorption $\left(\mathrm{EW}_{\mathrm{H} \alpha, \text { abs }}\right)$ and (ii) the stellar velocity corresponding to each spaxel in the data cube by anchoring the stellar populations models to the ones obtained for the LR $-V$ grating. The latest calculation requires us to reference the $\mathrm{HR}-R$ observations to the $\mathrm{LR}-V$ ones. Due to pointing effects, the spatial coverage of the galaxy is slightly different in both setups, so we have created data cubes with a pixel scale of 0 " 4 in each of the spatial axes. The area of these new synthetic spaxels, on which we will perform the subsequent analysis, will therefore be $0.4 \times 0.4 \operatorname{arcsec}^{2}$ and will be simply referred to as spaxels. The value of the spaxel size was chosen as the best compromise between the seeing of the observing nights and the routine used to create the data cubes. To estimate the $\mathrm{EW}_{\mathrm{H} \alpha \text {,abs }}$ we used the CALIFA spectrum (3700-7000 $\AA$ ) integrated in the same area as the one in our MEGARA FoV data cube. To recover the best-fit model of the stellar continuum, we run the pPXF code. Finally, we have obtained the Gaussian profile corresponding to the stellar absorption that is added to the $\mathrm{H} \alpha$ emission flux in the $\mathrm{HR}-R$ data cube. The main assumption in this approach is that the model that best reproduces the stellar continuum is the same for all of the spaxels in the MEGARA data cube. As the mean value of the recovered stellar flux at the $\mathrm{H} \alpha$ wavelength is around 9\% of the total $\mathrm{H} \alpha$ flux, we applied this correction assuming that it represents a small second-order correction for the estimation of the total $\mathrm{H} \alpha$ flux. As is done in the case of the $\mathrm{LR}-V$, we also increase the $\mathrm{S} / \mathrm{N}$ ratio, making a spatial binning of the data cube. In this case, we have defined the $\mathrm{S} / \mathrm{N}$ as the ratio between the $\mathrm{H} \alpha$ emission line and the nearest continuum and imposed an $\mathrm{S} / \mathrm{N}>15$.

The high spectral resolution of MEGARA allow us to distinguish double- and triple-peaked $\mathrm{H} \alpha$ emission-line profiles in this galaxy. The finding of up to three kinematically distinct gaseous components is in accordance with the results from Vega Beltran et al. (1997). Some examples showing the complexity of the $\mathrm{H} \alpha$ emission-line profiles will be shown and examined in the following sections.

\section{Results}

\subsection{Kinematics of the Emitting Gas as Revealed by the $H \alpha$ Emission Line}

To investigate the gas kinematics, we make use of the $\mathrm{H} \alpha$ emission line. A previous long-slit spectroscopic study of the kinematics of the gas in UGC 10205 was performed by Vega Beltran et al. (1997) using the Intermediate Dispersion Spectrograph at the Isaac Newton Telescope obtaining a mean value of $\mathrm{FWHM}=0.86 \AA\left(\sigma \sim 17 \mathrm{~km} \mathrm{~s}^{-1}\right)$. These authors found line splitting for three kinematically distinct gaseous components in the position-velocity diagram along the major axis of the galaxy in the inner $\pm 13^{\prime \prime}$. A double-peaked $\mathrm{H} \alpha$ emission appeared in the region $-13^{\prime \prime} \leqslant r<3^{\prime \prime}$ and a third $\mathrm{H} \alpha$ component is found at distances in the range $3^{\prime \prime} \leqslant r \leqslant 13^{\prime \prime}$. Later, García-Lorenzo et al. (2015) presented a comprehensive analysis of the gas velocity fields for 177 galaxies among which UGC 10205 is present. The galaxies were observed as part of the CALIFA survey using the Potsdam Multi-Aperture Spectrophotometer (PMAS; Roth et al. 2005) in the PPak mode (Kelz et al. 2006), which provides a nominal resolution $(R \sim 850$ at $\lambda \sim 5000 \AA$ ) that is not sufficient to probe the presence of multiple components in this object. 
Here, the high-resolution MEGARA IFS data improves the ability to map the kinematics of the line-emitting gas in a spatially resolved manner, combining the information on the morphology and kinematics simultaneously. In the next section, we attempt an interpretation for the kinematic properties of the ionized gas phase by fitting a rotating exponential thin-disk model to the observations.

\subsubsection{Thin-disk Model}

Both García-Lorenzo et al. (2015) and Vega Beltran et al. (1997) derived monotonically rising rotation velocity curves for the inner regions of UGC 10205. Following these results, we adopt an arctangent parameterization with a smooth turnover to recover the shape of the rotation curve, $V_{r}=(2 / \pi) v_{\max }$ $\arctan \left(r / r_{t}\right)$ (Courteau 1997), where $v_{\max }$ is the asymptotic maximum rotation speed and $r_{t}$ is the transition radius between the rising and flat part of the rotation curve.

A suite of 2D kinematic models is built to recover the 2D ionized velocity field of the purely rotating galactic disk. The models contain six free parameters: $v_{\max }, r_{t}$, position angle (PA), inclination ( $i$ ), and the position of the dynamical center $(x 0, y 0)$. To define the parameters' range, we use reported measurements and allow variations that are consistent with our data. The corresponding ranges are: $v_{\max }=(250-350) \mathrm{km} \mathrm{s}^{-1}$, $r_{t}=(0.05-0.5) \mathrm{kpc}, i=(20-89)^{\circ}$, and PA $=(40-170)^{\circ}$. For the dynamical center, we use the photometric center as our reference value. The photometric center is defined as the peak of the continuum emission in the region (6540-6620) $\AA$, and we allow a variation of $2^{\prime \prime}$ around it. A systemic velocity of $6556 \mathrm{~km} \mathrm{~s}^{-1}$ is taken from NED.

We utilize a minimization based on a mean-squared error to find the best fit between the model and data. As the $\mathrm{H} \alpha$ channels show a somehow clumpy distribution (see Figure 3), we apply the minimization on the voronois that are more relevant to the shape of the rotation curve. The model parameters that best describe the data are $v_{\max }=292 \mathrm{~km} \mathrm{~s}^{-1}$, $r_{t}=0.12 \mathrm{kpc}, i=75^{\circ}$, and $\mathrm{PA}=130^{\circ}$. In the case of the inclination, a wealth of values can be found in the literature. García-Lorenzo et al. (2015) derived a photometric inclination of $54^{\circ}$ using the whole galaxy, while Rubin et al. (1985) reported $i=84^{\circ}$. Due to the recent interactions suffered by the galaxy (as suggested in Reshetnikov \& Evstigneeva 1999), the gas disk of the outer parts might be mostly affected by them. Thus, the inclination for the innermost parts might be different. We have obtained $i=75^{\circ}$ for these regions. The orientation of the line of nodes is in reasonably good agreement with the value inferred by Barrera-Ballesteros et al. (2015). They derived a morphological $\mathrm{PA}=(132.4 \pm 1.6)^{\circ}$ by fitting an ellipse to an isophote at radius $15^{\prime \prime}$ in the $r$-band SDSS image. They also measured kinematic PA for the ionized component and derived $\mathrm{PA}=(120.3 \pm 2.7)^{\circ}$ and $\mathrm{PA}=(129.4 \pm 1.5)^{\circ}$ for the approaching and receding sides, respectively.

Figure 3 shows the $\mathrm{H} \alpha$ channel maps of the $\mathrm{HR}-R$ data cube. Each map represents the line emission at a particular wavelength channel (from 6705.0 to $6709.5 \AA$ ) with a channel step of $\Delta \lambda=0.5 \AA$. From the analysis of this figure, we infer the presence of a highly inclined gas-rich disk located in the central regions of this galaxy. The isovelocity contours are well reproduced by our model for the central parts (see the white solid lines in the channels maps from 6707.0 to $6708.5 \AA$ of Figure 3). However, some of the ionized gas do not follow the general rotation pattern, as indicated by the isovelocity curves (see the green and orange crosses in the channel maps from $\lambda=6704.5$ to $\lambda=6707.5 \AA$ in Figure 3 ). This component will be described later.

To recover the velocity maps of the ionized gas components, we need to disentangle the different emission lines in the $\mathrm{H} \alpha$ profile. We fit multiple Gaussian components and a polynomial continuum to separate up to three kinematically distinct gaseous components. The corresponding ionized gas kinematic maps are shown in the top panel of Figure 4.

The first component (component 1) is the one associated with the thin-disk model. We trace this component based on the velocity associated with the centroid of each Voronoi cell in the disk model, i.e., we defined the Gaussian fit of this component as the closest one to the value of the velocity in the model. Panels (e) and (f) in the bottom part of Figure 4 show the best thin-disk model for component 1 and the residuals after subtracting the best thin-disk model. The latter map indicates that the residuals fluctuate around zero randomly, which confirms that the thin-disk model is a good approximation. The results by Vega Beltran et al. (1997) pointed out the existence of a second component with a similar rotation pattern to the previous one. The spatial extension of this component (component 2) is more limited to the central part of the galaxy, as can be seen in panel (b) of Figure 4. There is also a third component (component 3 ) with a velocity close to the systemic, ranging from -60 to $85 \mathrm{~km} \mathrm{~s}^{-1}$ relative to the systemic. This last component is spatially coincident with the regions previously characterized as deviations from the general rotation pattern (the green and orange crosses in Figure 3).

Finally, we select a few characteristic Voronoi cells to show the complexity of the emission-line profiles together with the Gaussian fits of the three kinematically distinct components. The position of these Voronoi cells is shown with blue and orange colors in the middle panel of the top row in Figure 5. The blue, red, and orange Gaussian fits in the spectra of this figure correspond to components 1, 2, and 3 in Figure 4, respectively. The blue dashed line is the velocity of the thindisk model, while the orange dashed line represents the systemic velocity. Also, a significant rotational broadening for the $\mathrm{H} \alpha$ profiles in the central regions can be seen in this figure (see panel corresponding to voronoi $=3$ ). As we will describe later in Section 5.4, this effect is due to the steep rise of the rotational velocity curve in the innermost regions of this galaxy. We would expect the internal dynamics of these regions to be better constrained in nearly face-on or less inclined systems where the $\sigma$ values are expected to be less broad by the effect of rotation. In spite of the complex structure that follows the ionized gas distribution, our thin-disk model is able to reproduce the kinematic pattern found in this object.

\subsubsection{Deviations from the Pure Thin Disk Model}

As mentioned before, the distribution of the ionized gas in the low-velocity channels (from 6704.5 to $6707.5 \AA$ ) reveals the presence of a kinematically distinct component that does not follow the rotation pattern of the gas-rich disk. To explain the gas velocity field in those particular regions, two possible interpretations arise.

On one hand, this dynamical component seems to be spatially coincident with the position of the dust lanes that might be driving the motions of the gas (see positions marked as green and orange crosses in Figure 3). Dust lanes are, in most cases, morphologically associated with a gas dense 


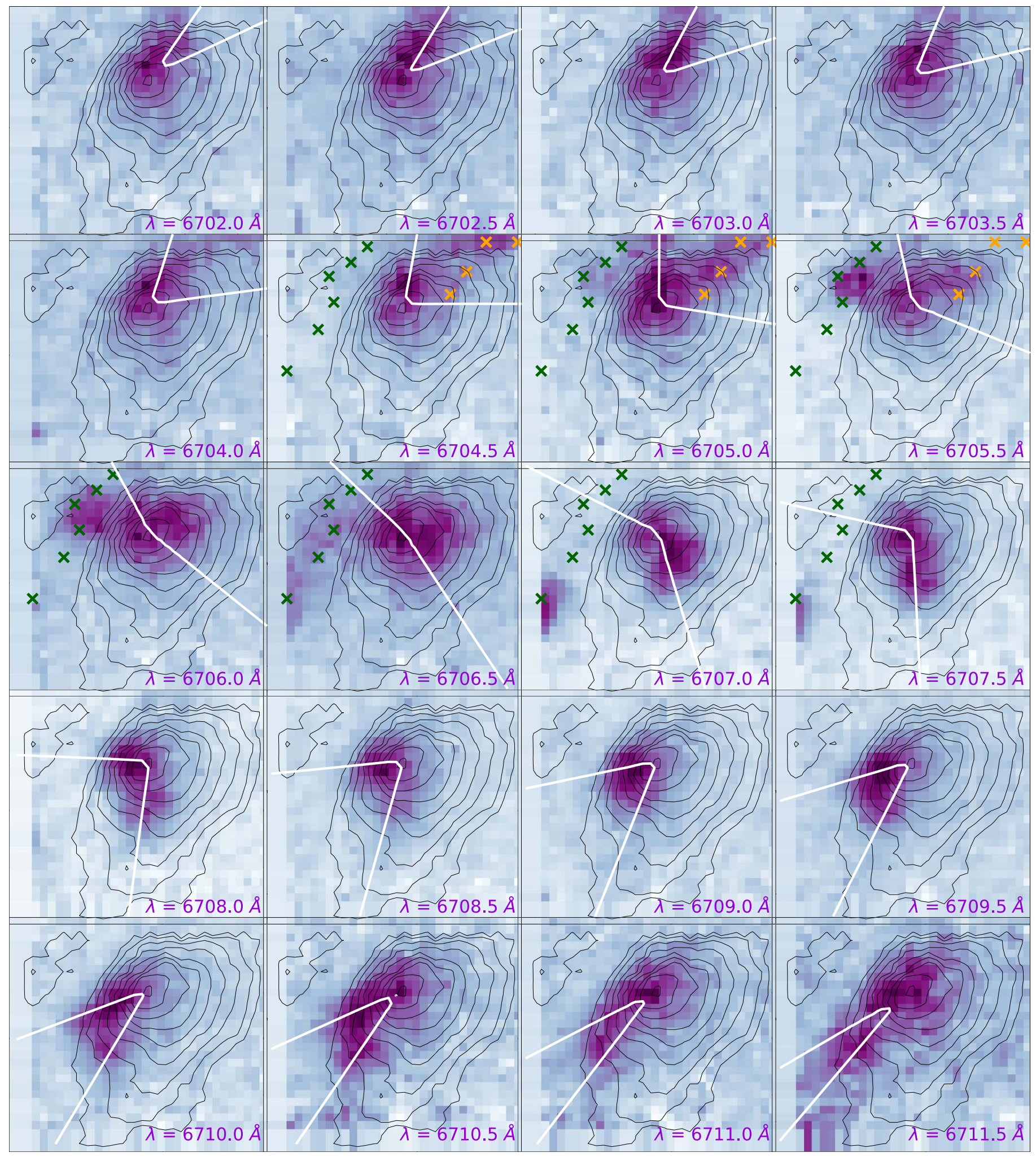

Figure 3. $\mathrm{H} \alpha$ velocity channel maps for the central part of the galaxy UGC 10205 in the velocity range $6363.14-6797.40 \mathrm{~km} \mathrm{~s}^{-1}$. A velocity bin of $22.86 \mathrm{~km} \mathrm{~s}^{-1}$ corresponding to a $\Delta \lambda=0.5 \AA$ is used to slice the data cube. The wavelength of each channel is shown in the lower right corner. White lines represent the isovelocity curves for a thin-disk model with inclination of $75^{\circ}$. Green and orange crosses represent the positions of the Voronoi cells that are best reproduced by a model with inclination of $83^{\circ}$ as discussed in Section 5.1.2. Continuum flux contours from the LR $-V$ data cube are shown as black solid lines.

component even in early-type galaxies (Finkelman et al. 2010, 2012). Judging from the large-scale images available for UGC 10205 (see upper panel in Figure 1 as an example), the well-defined and prominent dust lanes of this object are highly inclined. Due to the reduced size of the MEGARA FoV, we are sampling a relatively small angle on the sky in comparison with the large-scale size of the object $\left(\sim 1^{\prime}\right)$. Assuming a close to edge-on orientation for the dust lanes, we 

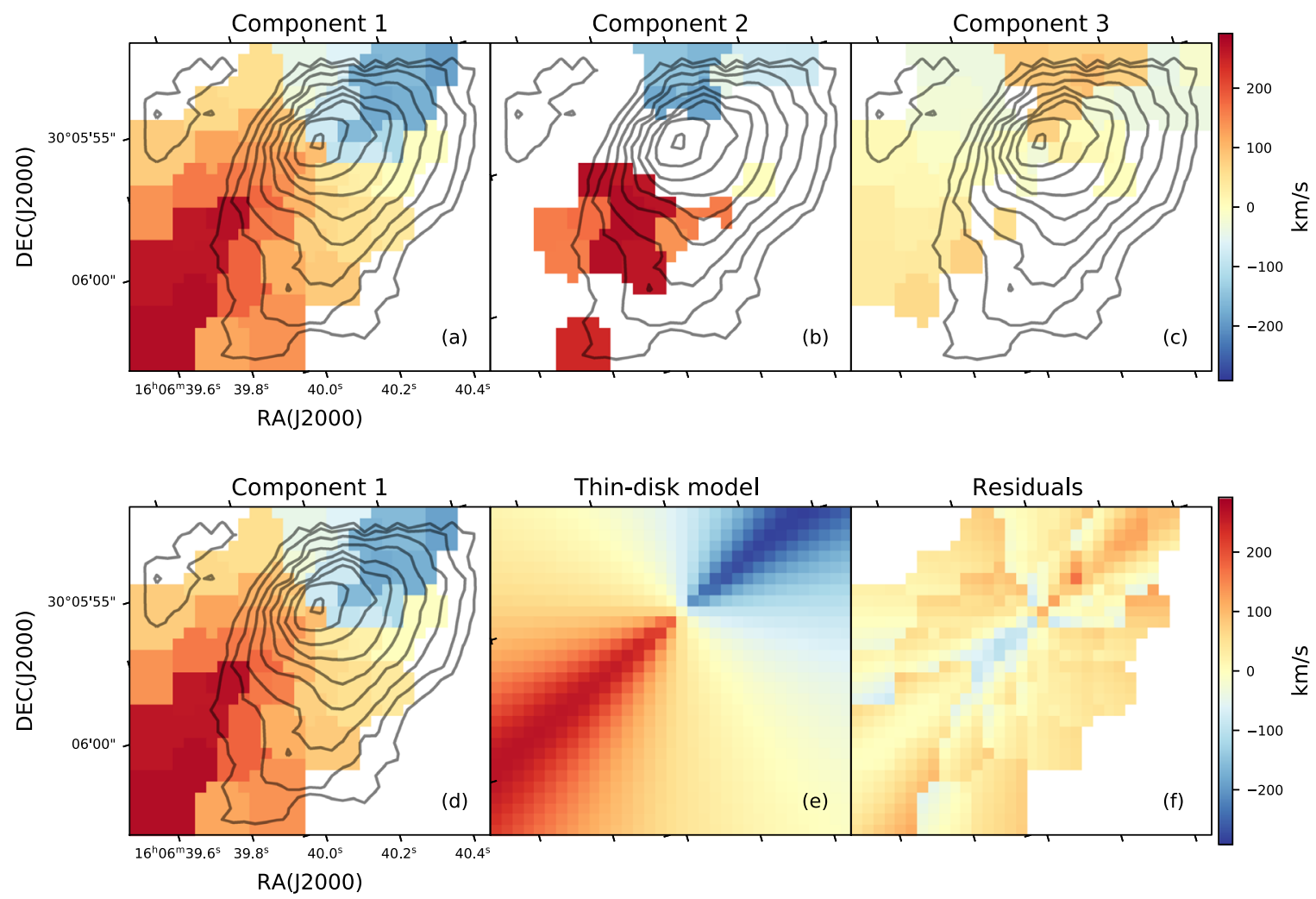

Figure 4. Top panel: velocity maps of the three kinematically distinct gaseous components: (a) component defined by the thin-disk model (arctangent function), (b) second component with a similar rotation pattern to the previous one, and (c) component with a velocity close to the systemic value. These components are defined by the blue, red, and orange Gaussian fits in the spectra of Figure 5, respectively. Continuum flux contours from the LR $-V$ data cube are shown as gray solid lines. Bottom panel: (d) velocity map for the ionized gas component defined by the thin-disk model, (e) the best thin-disk model for the previous component, (f) the residuals after subtracting the best thin-disk model.

expect to recover angles $(\phi)$ around $90^{\circ}$ and $270^{\circ}$ within this structure. Should that be the case, the difference between the radial velocity component of the gas in each Voronoi cell and the systemic velocity of the thin-disk model $\left(i=75^{\circ}\right)$ should be nearly zero. Possible values for the inclination of the dust lane were investigated to check whether or not the velocities in these regions might be coherent with the structure of a disk model with higher inclination than the main thin-disk model $\left(i=75^{\circ}\right)$. An optimal value of $i=83^{\circ}$ was found.

Thus, the bottom panel in Figure 6 shows the distribution of the distances and angles probed by the Voronoi cells in the plane of the dust lanes assuming $i=83^{\circ}$ for this plane. The SE (approaching) side of the dust lane is represented by the orange points, while the SW-to-NW (receding) side is marked by the green points. In particular, the points represent the centroid of each Voronoi cell associated with the regions where the line of sight passes through the plane of the dust lanes (positions also marked as green and orange crosses in Figure 3).

Additionally, the difference between the velocity of this gas component and the systemic velocity of the thin-disk model $\left(i=75^{\circ}\right)$ computed for each Voronoi cell is shown in the upper panel of Figure 6 as green/orange points. As expected, this difference in velocity $(\Delta v)$ is nearly zero for $\phi \sim 90^{\circ}$ and $\phi \sim 270^{\circ}$. As an example of a particular Voronoi cell, we include the spectrum corresponding to voronoi $=7$ (Figure 5). In this spectrum, two different features can be clearly seen: (i) a component almost at the systemic velocity of the model and (ii) the component of the thin-disk model. The former one would be the gas component associated with the dust-lane structure.
At the galactocentric distances corresponding to the positions of the receding side of the dust lane $\left(\sim 30^{\prime \prime}\right)$, we expect to reach the maximum velocity for the thin-disk model $\left(v_{\max }=292 \mathrm{~km} \mathrm{~s}^{-1}\right.$, green solid line). $i=83^{\circ}$ is the inclination of the dust-lane that minimizes the distance between the model and $\Delta v$. For the case of the SE side of the dust lane (orange points), the galactocentric distances are $\sim 10^{\prime \prime}$, so the velocity curve has still not reached its maximum velocity. A velocity of $130 \mathrm{~km} \mathrm{~s}^{-1}$ (orange dashed line) is the best value consistent with the inclination derived previously. Thus, having a slightly more inclined dust lane $\left(i=83^{\circ}\right)$ with the same galaxy's potential than the thin-disk model $\left(i=75^{\circ}\right)$ seems to be a reasonably good explanation for these regions.

On the other hand, the origin of this component could be associated with the merging scenario that creates a localized enhancement of the star formation with a distinct kinematic pattern, and the gas might be tracing non-circular motions in those regions. The numerical model presented by Reshetnikov \& Evstigneeva (1999) relied on the tidal disruption of a small E/SO object by the massive early-type galaxy. Non-circular gas motions in the nuclear region of UGC 10205 were suggested by these authors. However, the nice match between both the $\Delta v$ values and its azimuthal variation with respect to the one of highly inclined dust lanes seems to favor the former scenario.

\subsection{Resolved Stellar Kinematics}

The MEGARA absorption-line kinematics for the central regions of the galaxy UGC 10205 are presented in Figure 7. 

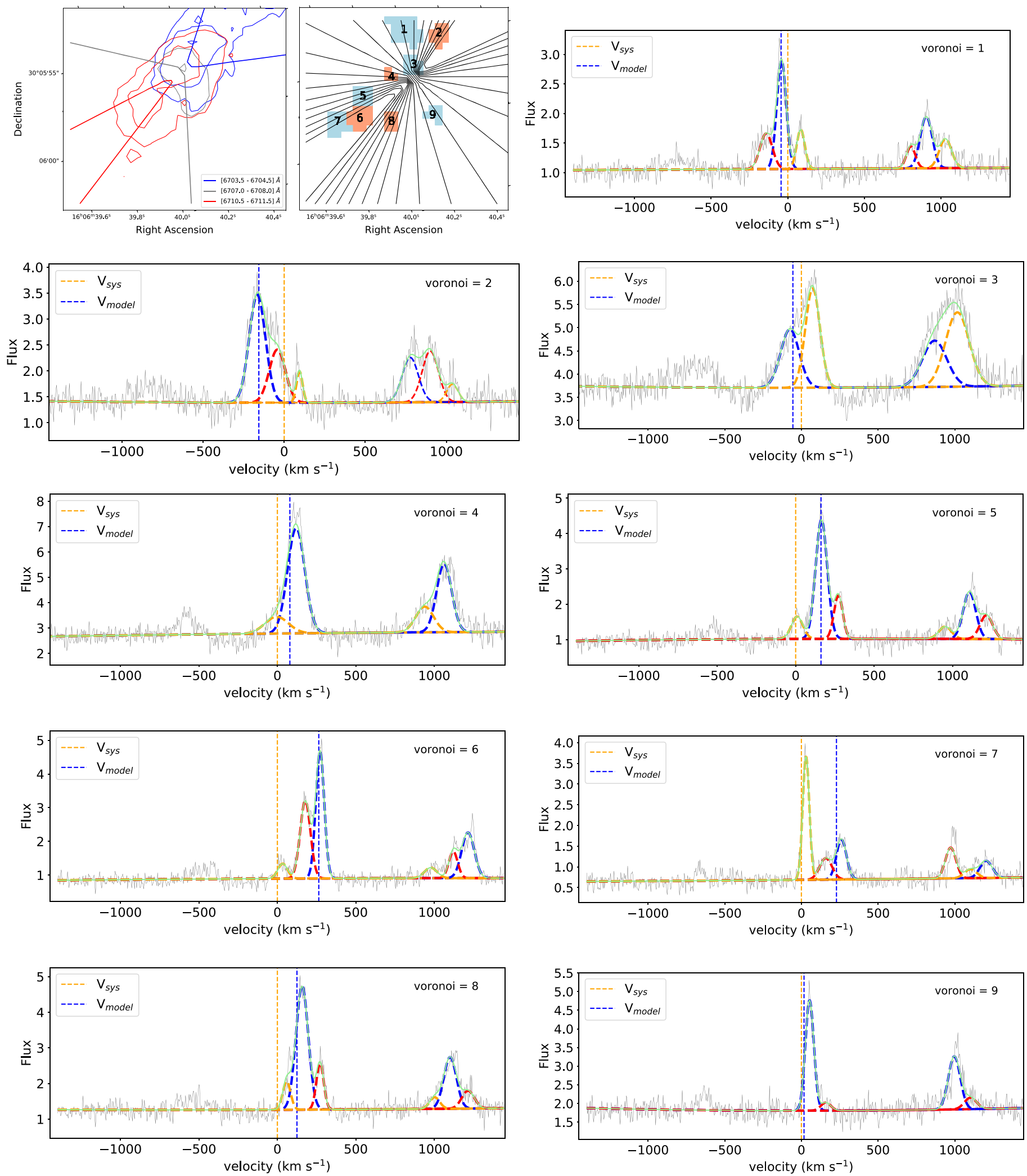

Figure 5. Left top panel: $\mathrm{H} \alpha$ flux contours together with the rotating thin-disk model derived for the $\mathrm{H} \alpha$ velocity field are shown as blue, gray, and red colors for ranges [6703.5-6704.5] $\AA$, [6707.0-6708.0] $\AA$, and [6710.5-6711.5] $\AA$, respectively. Middle top panel: isovelocity contours for the thin-disk model $\left(i=75^{\circ}\right)$ showing the location of some specific Voronoi cells within the data cube. The color coding assigned to the Voronoi cells is chosen for convenience, allowing for a better visual inspection of their spatial extent within the map. Remaining panels: examples showing the presence of kinematically distinct gaseous H $\alpha$ components for the Voronoi cells indicated in the middle top panel. The zero velocity is set at the systemic redshift of the galaxy (orange dashed line), while the velocity of the thindisk model for each particular Voronoi cell is shown by the blue dashed line. Gaussian fits represent the component associated with the thin-disk model (blue), the component with a similar rotation pattern to the previous one but restricted to a smaller region (red), and the component with a velocity close to the systemic (orange), respectively. The units of the horizontal axis are in $\mathrm{km} \mathrm{s}^{-1}$ relative to the systemic velocity of the galaxy. 


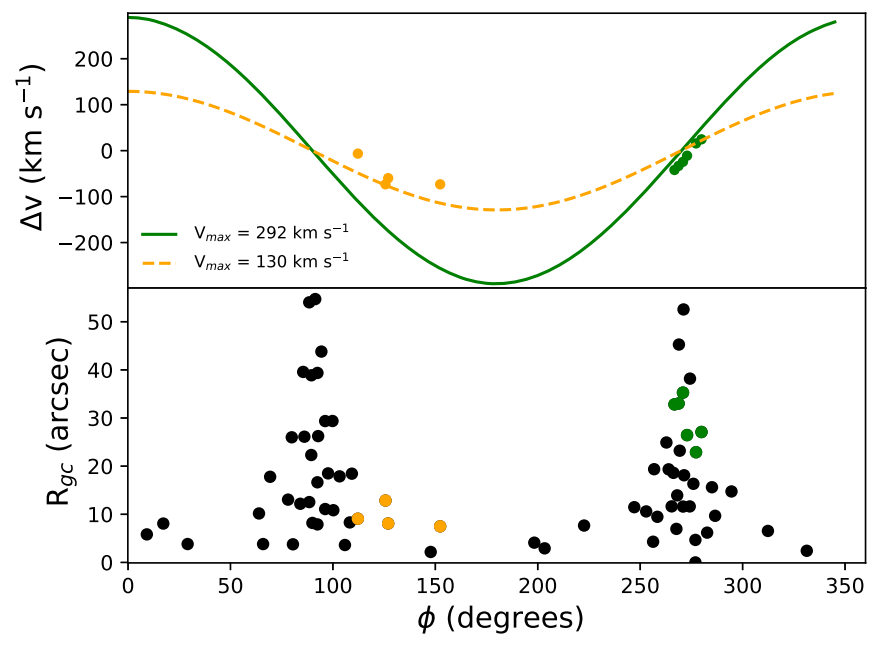

Figure 6. Top panel: velocity distribution in the plane of the dust lanes $\left(i=83^{\circ}\right)$. The orange (approaching side) and green (receding side) points represent the difference between the radial velocity component of the gas and the systemic velocity of the thin-disk model $\left(i=75^{\circ}\right)$ computed for each Voronoi cell $(\Delta v)$ within the dust lane. The green solid line and orange dashed line are the velocity models with $v_{\max }=292 \mathrm{~km} \mathrm{~s}^{-1}$ and $v_{\max }=130 \mathrm{~km} \mathrm{~s}^{-1}$, respectively. Bottom panel: distribution of the galactocentric distances and angles for the different Voronoi cells. The orange and green points represent the values of the ones located in the dust lane.
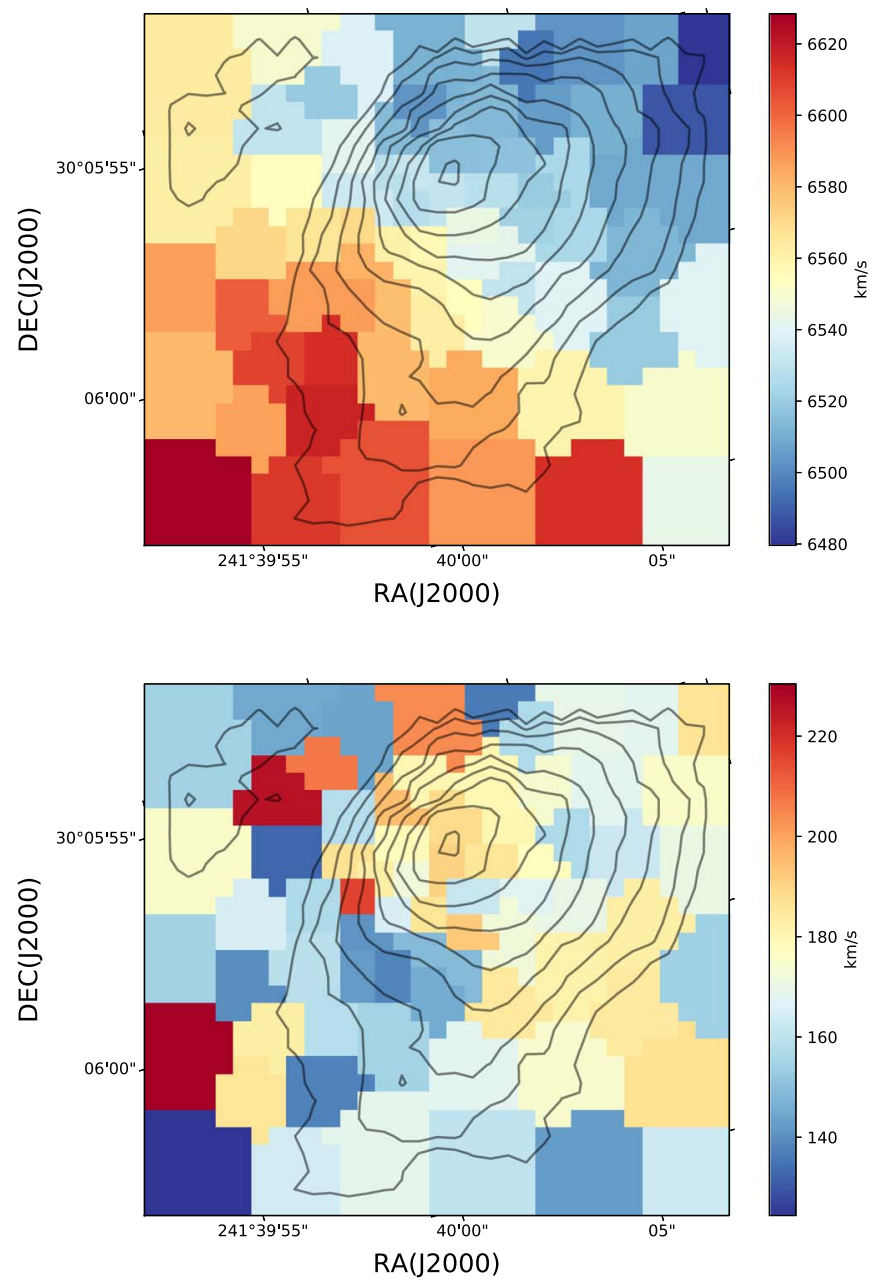

Figure 7. Top panel: stellar velocity field for UGC 10205. Note that the galaxy systemic velocity is reported to be $6556 \mathrm{~km} \mathrm{~s}^{-1}$. Bottom panel: stellar velocity dispersion. Contours of the continuum emission (black) are overlaid on both maps. Color scales and units are indicated on the right part of each map.
This figure shows both the stellar velocity field and the stellar velocity dispersion maps derived from our pPXF analysis. pPXF yields the values of the stellar mean velocity and stellar velocity dispersion for each Voronoi cell applying an $\mathrm{S} / \mathrm{N}$ rejection criterion of 20 . The reader is referred to Section 4.1 where the main procedure followed to create these maps is explained in detail.

The stellar velocity map (upper panel in Figure 7) shows a clear stellar rotation pattern with a symmetric velocity field with respect to the galaxy center and with a maximum radial velocity of $\sim 6628 \mathrm{~km} \mathrm{~s}^{-1}$. Typical errors in our stellar velocity measurements range from 4 to $23 \mathrm{~km} \mathrm{~s}^{-1}$ with mean errors of $10 \mathrm{~km} \mathrm{~s}^{-1}$. To check the accuracy of our kinematic analysis, a visual inspection is performed using the results presented in Falcón-Barroso et al. (2017). These authors found a similar stellar rotation pattern using the mid-resolution CALIFA data (V1200 with $R \sim 1650$ ) in the wavelength range 3850-4600.

The stellar velocity dispersion map (bottom panel in Figure 7) displays values ranging from 124 to $230 \mathrm{~km} \mathrm{~s}^{-1}$, with values in the nuclear region $\sim 190 \mathrm{~km} \mathrm{~s}^{-1}$. These values are already corrected for the instrumental resolution, which, in our case, is $\sigma \sim 21 \mathrm{~km} \mathrm{~s}^{-1}$. Although no signs of kinematically decoupled components appeared to be present, we have performed the analysis of the Gauss-Hermite moment $h 3$ (skewness of the velocity profile) to examine this effect. The $h 3$ map (not shown) indicates that we can safely discard the presence of an anticorrelation between these values and those of the stellar velocities.

\subsection{Estimating the Asymmetric Drift Correction}

By applying an asymmetric drift correction (ADC), it is possible to account for the effect of the stellar random motions within galaxies. Basically, the mean tangential velocity of a stellar population lags the actual circular velocity as defined by the gravitational potential of the galaxy. ADC accounts for this difference. The effect of the ADC phenomenon will be more significant in those cases where the velocity dispersion of different stellar populations is higher. Although this effect was first noted in the solar neighborhood (Strömberg 1924, 1925), ADCs have been extensively used in the literature to reconcile gas and stellar rotation curves observed in external galaxies.

The Stellar Velocity Ellipsoid (SVE) is described in cylindrical coordinates by its radial, tangential, and vertical components: $\sigma_{R}, \sigma_{\phi}$, and $\sigma_{Z}$. The shape of the SVE could be parameterized by the axial ratios $\sigma_{Z} / \sigma_{R}, \sigma_{\phi} / \sigma_{R}$, and $\sigma_{Z} / \sigma_{\phi}$. For the purpose of this work, we focus our attention on the $\alpha=\sigma_{Z} / \sigma_{R}$ and $\beta=\sigma_{\phi} / \sigma_{R}$ parameters. In particular, Mogotsi \& Romeo (2019) found a global vertical-to-radial velocity dispersion ratio of $\alpha=0.97 \pm 0.07$ for UGC 10205. Similar values of the radial and vertical dispersions point to an isotropic heating.

Here, we assume that the stellar azimuthal anisotropy $\left(\beta_{\phi}\right)$ can be approximated using the epicycle anisotropy, i.e., the stellar orbits are considered nearly circular (Gerssen et al. 1997, 2000; Shapiro et al. 2003; Ciardullo et al. 2004; Westfall et al. 2011; Gerssen \& Shapiro Griffin 2012; Blanc et al. 2013; Gentile et al. 2015). Thus, we measure $\beta_{\mathrm{EA}}$ (hereafter $\beta$ for simplicity) using the following expression:

$$
\beta=\sqrt{\frac{1}{2}\left(\frac{\partial \ln \left(v_{\phi}\right)}{\partial \ln (R)}+1\right)}
$$


where $v_{\phi}$ is the tangential speed of the stars. We found a mean value of $\beta=0.86 \pm 0.12$. Due to the small dispersion found and in order to simplify the calculations, we assume the previous value for all of the Voronoi cells.

Once $\alpha$ and $\beta$ are known, we infer $\sigma_{R}$ in each Voronoi cell using the values of $\sigma_{\text {LOS }}$ previously obtained (bottom panel of Figure 7):

$$
\sigma_{\mathrm{LOS}}^{2}=\sigma_{R}^{2} \sin \phi^{2} \sin i^{2}+\sigma_{\phi}^{2} \cos \phi^{2} \sin i^{2}+\sigma_{Z}^{2} \cos i^{2}
$$

where $\phi$ is the angle of the central position of each Voronoi cell in the plane of the galaxy measured from the line of nodes and $i$ refers to the inclination. We assume the same inclination for each Voronoi cell, i.e., in this case, we adopt the same inclination for the innermost regions of the galaxy. Due to the agreement between the ionized gas rotation axis and the stellar rotation axis with a difference of $(6 \pm 3)^{\circ}$, we use an inclination of $75^{\circ}$ for the ADC.

To estimate the ADC in each Voronoi cell, we apply the following expression as reproduced from equation (4-33) of Binney \& Tremaine (1987):

$$
\begin{aligned}
V_{C}^{2}= & v_{\phi}^{2}-\sigma_{R}^{2}\left[\frac{\partial \ln \left(\nu_{R}\right)}{\partial \ln (R)}+\frac{\partial \ln \left(\sigma_{R}^{2}\right)}{\partial \ln (R)}\right. \\
& \left.+1-\frac{\sigma_{\phi}^{2}}{\sigma_{R}^{2}}+\frac{R}{\sigma_{R}^{2}} \frac{\partial\left(v_{R} v_{Z}\right)}{\partial Z}\right] .
\end{aligned}
$$

This expression shows the difference between the true velocity (i.e., $V_{C}$ is the circular velocity of a test particle in the potential) and the observed circular velocity. If the SVE is aligned with the cylindrical coordinate system $(R, \phi, z)$, the last term in Equation (3) could be neglected.

The term $\nu_{R}$ in Equation (3) is proportional to the stellar mass surface density $(\Sigma)$ under the assumption that $\Sigma$ is well traced by the surface brightness profile of the galaxy. In this case, to trace the radial dependence of $\nu$, we apply a onedimensional (1D) photometric decomposition over the Panoramic Survey Telescope and Rapid Response System (PanSTARRS; Chambers et al. 2016) $y$-band light profile. Among all of the Pan-STARRS bands, the near-infrared one is considered the best tracer of the stellar mass as the light is mainly dominated by old MS and red giant branch stars. Also, problems associated with the interstellar dust (as shown by the presence of prominent dust lanes in UGC 10205) are partly avoided. The fit was performed following the procedure explained in Dullo et al. (2019). We discard non-symmetric features due to bars or spiral arms in the fitting process. The bulge component is parameterized using a Sérsic profile (Sérsic 1968) with a best-fitting Sérsic index $n=1.48 \pm 0.22$ and a half-light radius $r_{e}=4$ !" $10 \pm 0$ ". 81 . The galaxy disk component is described with an exponential profile with scale length $h=10$ ". $8 \pm 1$ !" 3 .

\subsection{Asymmetric Drift Correction: Comparison with Previous Data in the Literature}

Figure 8 shows the results obtained after applying the ADC to the stellar component. For comparison, the predictions for the rotation curves of Sérsic bulges obtained by Noordermeer (2008) are also shown. These curves have been scaled up to take into account the mass and the effective radius of the bulge

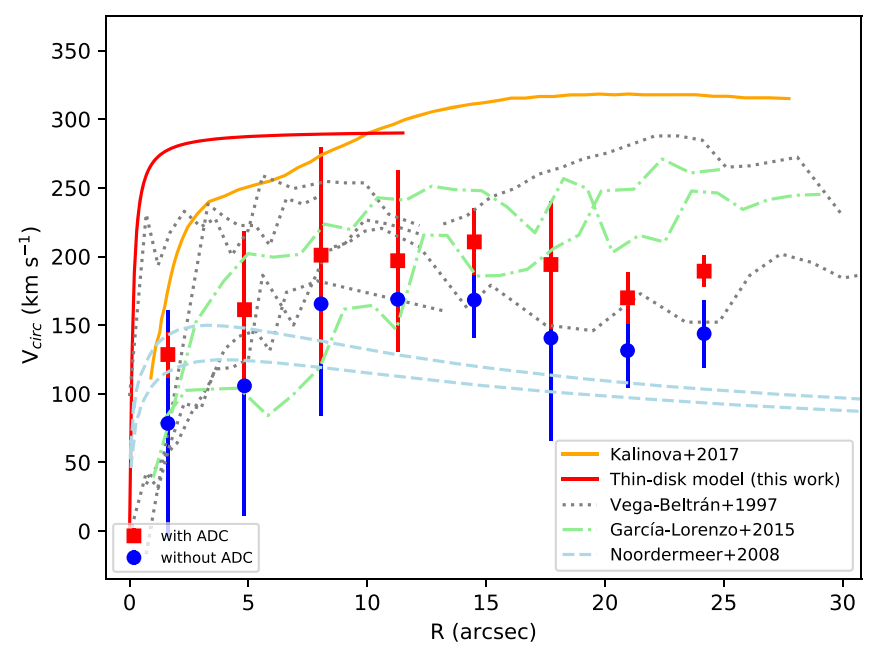

Figure 8. Stellar (blue circles) rotation curve for UGC 10205. The ionized gas rotation curves (red squares) after applying the ADC under the assumption of an inclination $i=75^{\circ}$. The light-blue dashed lines are the predictions for the rotation curves of a Sérsic bulge with $n=2$ and different values of the axis ratio $q=0.2$ (top) and $q=1$ (bottom) derived by Noordermeer (2008) and scaled up to match the mass and the effective radius of our bulge component. The best rotation thin-disk model is represented by the red solid line. The observed velocity curve values from Vega Beltran et al. (1997) and GarcíaLorenzo et al. (2015) are shown by the dotted gray lines and dotted-dashed green lines, respectively. The velocity curve profile measured by Kalinova et al. (2017) is also shown (orange line).

component. The effective radius, obtained directly from our 1D decomposition, is $r_{e}=4$ !" $10 \pm 0$ !" $81\left(r_{e} \sim 1.97 \mathrm{kpc}\right)$. For the stellar mass derivation, we apply the bulge-to-total flux ratio $(\mathrm{B} / \mathrm{T}=0.19 \pm 0.3)$ obtained as a result of the $1 \mathrm{D}$ decomposition and the total stellar mass of the galaxy derived in Walcher et al. (2014) $\left(\log \left(M_{\star}\right)=10.997 \pm 0.111 M_{\odot}\right)$. As the $1 \mathrm{D}$ decomposition yields $n=1.48 \pm 0.22$, we represent the rotation curve for a fiducial bulge with a Sérsic concentration parameter $n=2$ as the best approximation in our case. For the same $n=2$, the blue dashed line curves correspond to variations of the axis ratio $q=0.2$ (top) and $q=1$ (bottom) in Figure 8. It is expected that the rotation curves from Noordermeer (2008) are a lower limit, as they only consider the bulge mass component, but our $V_{C}$ measurements also trace the mass in the inner disk of the galaxy.

We have also included in Figure 8 some observational data found in the literature for this galaxy. In particular, the stellar rotation curve derived in Kalinova et al. (2017) is shown in orange. In the new classification proposed by these authors, the rotation curve of UGC 10205 is classified as round-peaked, meaning that "the circular velocity rises steeply and has a round peak, gradually changing to the flat part of the circular velocity curve (CVC) at radius $\sim 0.5 R_{e}$," where $R_{e}$ is the value they derived for the whole galaxy. They also found that this class is the most common one among early-type galaxies (E4-S0a) and spiral galaxies classified as $\mathrm{Sa}-\mathrm{Sbc}$. The typical value expected for the average amplitude of the CVC is around $300 \mathrm{~km} \mathrm{~s}^{-1}$, similar to the $v_{\max }$ value derived in Section 5.1 for the thin-disk model.

On the other hand, measurements of the ionized gas velocity rotation curve are also included. As the thin-disk model derived in Section 5.1 was recovered using mainly the inner regions of the galaxy, we include here other literature data that cover the entire galaxy. The velocity curve based on the $\mathrm{H} \alpha+[\mathrm{NII}]$ 
emission-line data obtained by García-Lorenzo et al. (2015) within CALIFA appears as the green dotted-dashed lines in Figure 8. The UGC 10205 major-axis kinematic curves derived by Vega Beltran et al. (1997) for the multiple $\mathrm{H} \alpha$ components found on this object using long-slit spectroscopy data are shown by the gray dotted lines. Both García-Lorenzo et al. (2015) and Vega Beltran et al. (1997) derived monotonically rising rotation velocity curves for the inner regions of UGC 10205. These data have been homogenized to $i=75^{\circ}$. Our stellar data points once corrected by ADC effects are in good agreement with the values obtained by the previous authors up to a galactocentric distance of $R \sim 17^{\prime \prime}$. For $R>17^{\prime \prime}$, our measurements match the ones by Vega Beltran et al. (1997) along the NW side of the major axis of the galaxy. These results suggest that assuming an inclination of $75^{\circ}$ for the stellar component in the central part of this galaxy was a reasonable approach. Also, the adoption of a tangential parameterization with a smooth turn-over to recover the shape of the thin-disk model in Section 5.1 is justified by the shape of the previous rotation curves.

Average values of the ADC in local disk galaxies are usually $10 \%-20 \%$ in late-type systems (see Ciardullo et al. 2004; Merrett et al. 2006; Noordermeer et al. 2008; Herrmann \& Ciardullo 2009; Westfall et al. 2011; Martinsson et al. 2013). Higher values for the ADC are expected for E/S0 and early-type spirals. Here, we are analyzing the central part of an S0/Sa galaxy. We found a mean value of $V_{\text {rot }}($ stars $) / V_{\text {rot }}$ (gas) $=0.75 \pm 0.08$. This result is consistent with the one by Cortese et al. (2014). These authors explored only the central parts of a sample mainly composed by early-type spirals finding an average ratio of $V_{\text {rot }}($ stars $) / V_{\text {rot }}$ (gas $)=0.75$. These results point out the necessity of having high values of the ADC in order to reconcile the discrepancy between the ionized and the stellar velocity rotation curves for these morphological types.

\subsection{Nature of the Absorbing Gas through ISM Na I D Measurements}

Galactic winds are an essential element that determines the evolution of galaxies through cosmic time. In particular, as explained in the introduction, galactic outflows are thought to play a key role in self-regulating star formation in galaxies, and they also constitute necessary mechanisms to explain the chemical evolution and metal enrichment of the circumgalactic medium (Oppenheimer \& Davé 2006, 2008).

At optical wavelengths, the ISM Na I D absorption doublet is commonly used to trace the properties of neutral atomic gas inflows and outflows in the ISM (Heckman et al. 2000; Schwartz \& Martin 2004; Martin 2005, 2006; Rupke et al. 2005; Veilleux et al. 2005; Chen et al. 2010; Sarzi et al. 2016; Roberts-Borsani \& Saintonge 2019). Due to the high inclination of this object, the NaI D absorption-line profiles are confused with multiple components along the line of sight. Searching for signatures of inflowing/outflowing material in highly inclined systems require having high spatial and spectral-resolution observations to break up the absorption profile into different components. Here, the observations provided by MEGARA show the variety of the profiles and the distinct kinematic components for the $\mathrm{Na}$ I D excess. Some examples of the ISM absorption Na I D lines that will be explained later (Sections 5.5.1 and 5.5.2) are provided in Figure 9.
For the discussions about inflows/outflows that appear in the following sections, we assume that the foreground gas is the dominant source of the absorptions found in the $\mathrm{Na} I \mathrm{D}$ profiles. In this scenario, blueshifted absorption is a signature of gas moving toward the observer (outflow) while redshifted absorption is indicative of gas moving toward the galaxy (inflow). We do not detect re-emission of absorbed photons from the background gas. For more details about this topic, the reader is referred to the detailed discussion found in RobertsBorsani \& Saintonge (2019).

\subsubsection{Infalling Neutral Gas}

The analysis of the previous profiles allows us to capture the presence of a redshifted high-velocity component (see the left panel of Figure 9) that might be associated with material moving toward the galaxy center. In particular, the $\Delta V$ distribution of the redshifted narrow $\mathrm{NaI} \mathrm{D}$ continuum absorption features shows a positive mean value of $\Delta V=(108.35 \pm 18.05) \mathrm{km} \mathrm{s}^{-1}$ relative to the systemic velocity of the galaxy. This redshifted component is located all over the MEGARA FoV. Also, the small variation of the $\Delta V$ distribution among all of the Voronoi cells is an indication that the infalling neutral gas extends beyond the spatial coverage of the FoV.

The previous value suggests a scenario for the ISM Na I D absorption lines in which the redshifted $\mathrm{Na}$ I D interstellar gas is moving inward toward the central regions of UGC 10205. Thus, the absorbing neutral material would be located in a shell-like spatial distribution region moving toward the galaxy as an inflowing gas. We consider this structure to be a shell-like type rather than a stream, based mainly upon the following two arguments. From a morphological point, there is a complete 2D coverage of the FoV. In the case of the shell, a 2D coverage is expected while the stream structure would be seen as a strip of stars or gas. From a kinematical point of view, in the current scenario where the bulk velocity of the inflowing gas presents almost a constant value (approximately $108 \mathrm{~km} \mathrm{~s}^{-1}$ relative to the systemic velocity of the galaxy) instead of showing a velocity gradient, we consider that the most simple explanation would be to have roughly spherically symmetric gas inflows. The current stage of this galaxy should be highly affected by the previous minor merger suffered in the past as proposed in Reshetnikov \& Evstigneeva (1999). The presence of these gas clouds that are inflowing toward the center of the galaxy might be a consequence of such scenario.

\subsubsection{Presence of Outflow Signatures: Mass and Outflow Rate of the Wind}

Major galaxy mergers and ULIRGS in the local universe exhibit outflows with relatively large velocities and a conical shape (Rupke et al. 2002; Rupke \& Veilleux 2005; Soto et al. 2012; Westmoquette et al. 2012; Cazzoli et al. 2014; Perna et al. 2019). Here, we present results from a less active starforming galaxy. The right panel in Figure 9 shows that clear blueshifted Na I D absorption signatures are present in this galaxy. The spectra clearly show the presence of doublepeaked components in the $\mathrm{Na}$ I D absorption-line profiles. High spectral resolution is needed to distinguish these components. The analysis of the spatially resolved optical spectroscopy of the blueshifted NaI D suggests the presence of a wide-angle conical outflow emerging from the central regions of UGC 

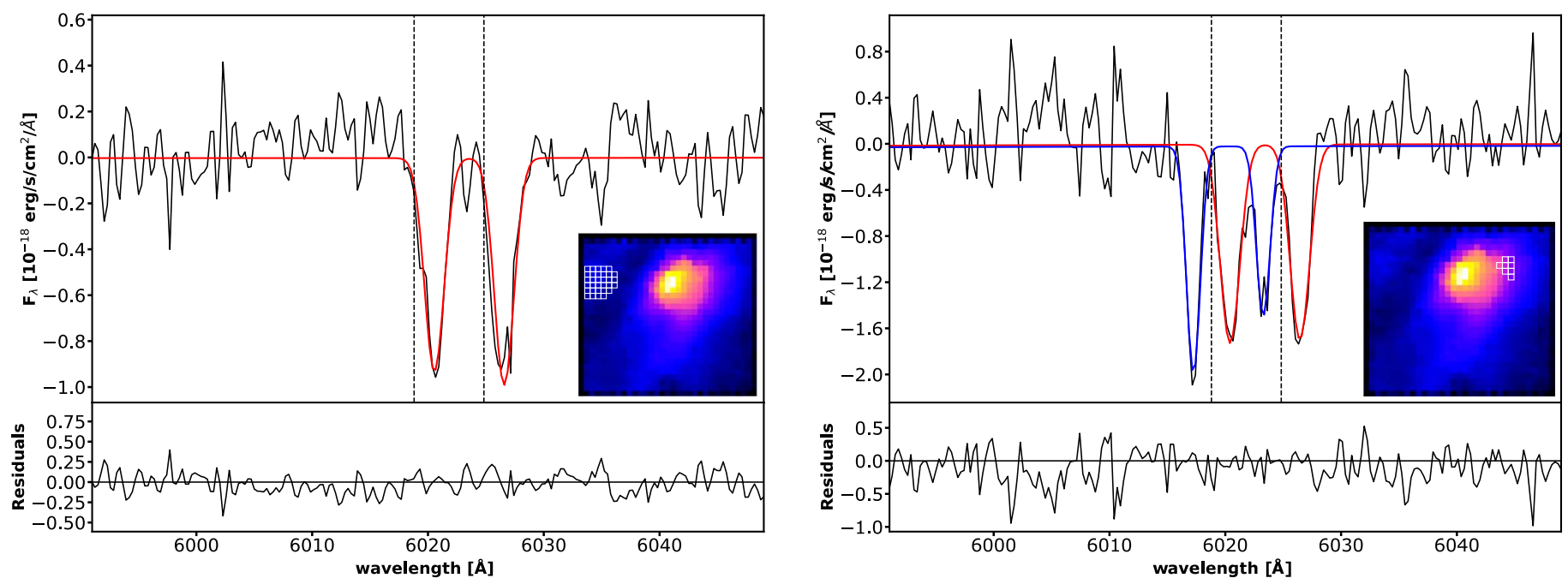

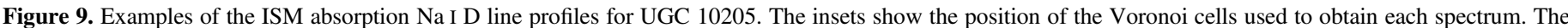

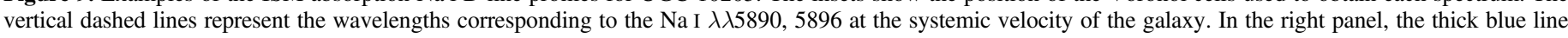

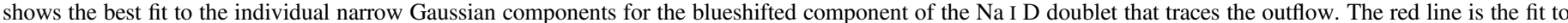

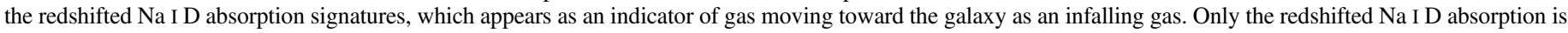
present in the left panel.

10205 and perpendicular to the galactic plane (Figure 10). The outflow shows a maximum projected velocity of up to $-87 \mathrm{~km} \mathrm{~s}^{-1}$, blueshifted with respect to the systemic.

To explore to what extent this neutral gas outflow might remove relatively large amounts of gas from the galaxy, we estimate both the total mass in the wind and the outflow mass rate applying the thin-shell free wind model (Equations (13) and (14) in Rupke et al. 2005). Since we have information on the spatial distribution of the wind thanks to the IFS data, we follow the approach of Shih \& Rupke (2010), Rupke \& Veilleux (2013), and Cazzoli et al. (2014, 2016) using a Voronoi-by-Voronoi basis and assuming a series of thin shells to make it a valid approach for this case. Following the previous prescription, the outflow mass rate is proportional to the deprojected velocity, the HI column density, and the deprojected solid angle measured in each Voronoi cell. The prescriptions applied to convert $\mathrm{Na}$ column densities into $\mathrm{H} \mathrm{I}$ column densities are largely affected by the assumptions made on the ionization fraction, the depletion onto dust grains and the $\mathrm{Na}$ abundance. To avoid the previous uncertainties in the estimation of the column density of $\mathrm{HI}$ atoms, we adopt here the same approach as in Cazzoli et al. (2014, 2016). These authors make use of both the relation between the $\mathrm{H}$ I and the color excess proposed by Bohlin et al. (1978) $\left(N_{\mathrm{HI}}=4.8 \times\right.$ $\left.E_{(B-V)} \times 10^{21} \mathrm{~cm}^{-2}\right)$ and the relation between the color excess and the equivalent width as in Turatto et al. (2003) $\left(E_{(B-V)}=\right.$ $\left.-0.04+0.51 \times \mathrm{EW}_{\mathrm{NaD}}\right)$. We calculate a mean value of $1.65 \pm$ $0.07 \times 10^{21} \mathrm{~cm}^{-2}$ for the $\mathrm{H} \mathrm{I}$ column density that is in the range of the expected values for low- $z$ galaxies as reported in Heckman et al. (2015) and Martín-Fernández et al. (2016). The cold-gas mass outflow rate is $0.78 \pm 0.03 M_{\odot} \mathrm{yr}^{-1}$, and the total mass in the wind is equal to $4.55 \pm 0.06 \times 10^{7} M_{\odot}$. These values are consistent with the ones derived using the formalism of the thinshell model in galaxies with lower star formation rates (SFRs), as in Roberts-Borsani \& Saintonge (2019) where the authors used galaxies with SFRs in the range $1.45<\mathrm{SFR}\left[M_{\odot} \mathrm{yr}^{-1}\right]<$ 16.98 , obtaining outflow mass rates ranging from $0.15<\dot{M}_{\text {out }}$ $\left[M_{\odot} \mathrm{yr}^{-1}\right]<1.74$.

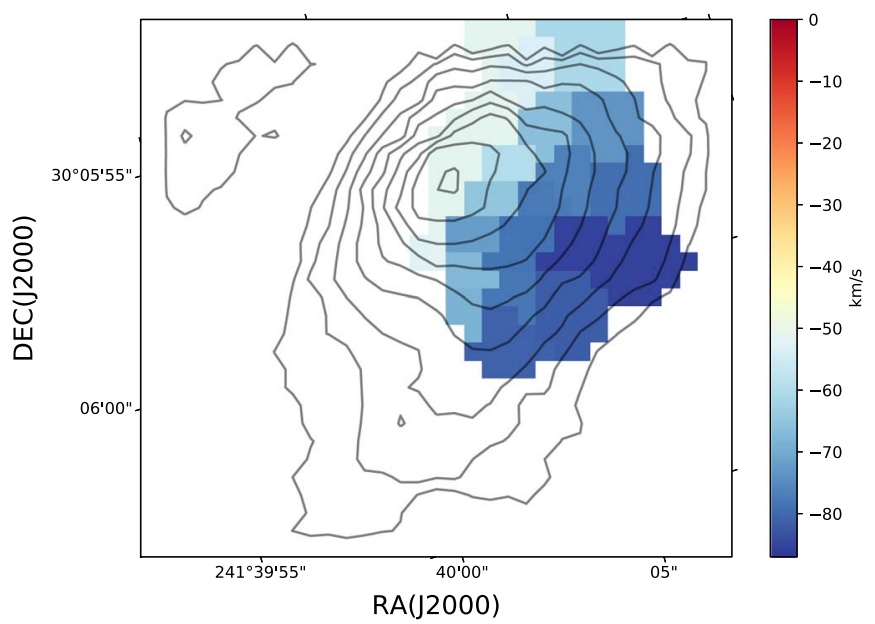

Figure 10. Neutral gas kinematics derived for the blueshifted absorption component of the $\mathrm{Na}$ I $\mathrm{D}$ doublet with respect to the systemic velocity of the galaxy as inferred from the MEGARA data. The contours indicate the distribution of the continuum flux from the $\mathrm{LR}-V$ data cube.

A significant mass loss has been postulated to regulate the star formation processes within galaxies. The mass loading factor, expressed as the ratio $\eta=\dot{M}_{\text {out }} / \mathrm{SFR}$, is used to describe the strength of the winds. This factor has important consequences for the predictions in the theoretical models, with some findings suggesting that $\eta$ increases with redshift (Barai et al. 2015; Muratov et al. 2015; Sugahara et al. 2017). To calculate the SFR, we have derived dust-corrected $\mathrm{H} \alpha$ growth curves using CALIFA data (following the methodology explained in detail in Catalán-Torrecilla et al. 2017). In the region covered by the MEGARA FoV and applying the conversion $\operatorname{SFR}(\mathrm{H} \alpha)=5.5 \times 10^{-42} L\left(\mathrm{H} \alpha_{\text {corr }}\right)$ (Kennicutt et al. 2009), we obtained $\operatorname{SFR}\left(\mathrm{H} \alpha_{\text {corr }}\right)=0.49 M_{\odot} \mathrm{yr}^{-1}$ and $\eta=1.59$. Due to the large amount of dust present in this galaxy, the attenuation correction based on Balmer Decrement measurements might be underestimating the SFR and affecting the real value of $\eta$. To mitigate this problem, we make use of 
hybrid calibrations combining luminosities measured directly with that of the light emitted by dust after being heated by young massive stars (Gordon et al. 2000; Inoue et al. 2001; Iglesias-Páramo et al. 2006; Calzetti et al. 2007; Hao et al. 2011; Kennicutt \& Evans 2012; Catalán-Torrecilla et al. 2015). We adopt the $\mathrm{H} \alpha_{\text {obs }}, \mathrm{FUV}_{\text {obs }}, 22 \mu \mathrm{m}$ and TIR luminosities calculated for this object in Catalán-Torrecilla et al. (2015) as our best estimations. Using the hybrid SFR prescriptions for the different combinations of $\mathrm{H} \alpha+a \times \mathrm{IR}$ and FUV $+b \times \mathrm{IR}$, we derived a mean SFR of SFR $=1.32 M_{\odot} \mathrm{yr}^{-1}$ and a loading factor of $\eta=0.59$. As these SFR measurements are for the whole galaxy while the effect of the outflow has been computed at a restricted distance limited to our current FoV, the estimated value of $\eta$ serves here as a lower limit. Mass loading factors below unity are typically found on galaxies similar to UGC 10205 (see Roberts-Borsani \& Saintonge 2019). Large-scale observations that trace the wind extension outside the disk are needed to explore the effect in the neighboring ISM and circumgalactic medium.

\section{Discussion: Proposed Scenario to Understand the Complex Kinematics in UGC 10205}

UGC 10205 was proposed as a possible candidate for being a polar-ring galaxy (Whitmore et al. 1990; van Driel et al. 2000). Further observations made with CALIFA revealed that there is not a clear misalignment between the velocity maps of the gas (García-Lorenzo et al. 2015) and the stellar components (Falcón-Barroso et al. 2017). As seen in the 2D stellar kinematics maps of Figure 7, the approaching radial velocities are on the SE side of the galaxy, which means the rotation of the disk is counterclockwise.

Dust lanes appeared roughly parallel to the major axis of the galaxy. In particular, the one that is crossing the southern region is more clearly visible in front of the bulge (i.e., on the near side of the galaxy) while the one in the north seems to be hidden behind the bulge. This location within the galaxy is consistent with the orientation of the reported outflow, which is seen over the near side of the galaxy. As the cold neutral gas traced by $\mathrm{NaI} \mathrm{D}$ needs to be observed against the stellar background, the outflow is detected in the approaching cone, while the receding part of the cone is not observed. The dust lane that is crossing the galaxy in the south position would prevent us for seeing the outflow component arising in this region, which suggest that the dust lane is located on the near side of the galaxy from our position. On the contrary, the dust lane in the north would be located on the far side of the galaxy.

To elucidate the possible mechanism that originated the outflow, it is important to discriminate between different ionization sources in the nuclear region. In Catalán-Torrecilla et al. (2015), we applied the standard Baldwin, Phillips, and Terlevich diagram (Baldwin et al. 1981) with the demarcation lines of Kauffmann et al. (2003) and Kewley et al. (2001). In particular, we estimated the $[\mathrm{O} \mathrm{III]} / \mathrm{H} \beta$ and the $[\mathrm{N} \mathrm{III}] / \mathrm{H} \alpha$ line flux ratio for the central $3^{\prime \prime}$ spectra using the CALIFA IFS data. The results pointed out that the center of this galaxy could be classified as a mixed SF/AGN region, i.e., the points lied on the composite area of the diagram. This analysis discards the presence of a strong AGN contribution. This is consistent with the studies by Sarzi et al. (2016) where the authors examined a sample composed mostly of massive early-type galaxies suggesting that the cold-gas outflows typically occur in objects dominated either by central starbursts or composite AGN/star formation activity. The spatial distribution of the $\mathrm{H} \alpha$ emission shows that there is a strong resolved $\mathrm{H} \alpha$ contribution in the nuclear region (see the channel maps presented in Figure 3). This might favor a scenario where star formation activity could be the potential mechanism triggering the Galactic wind. The presence of the outflow is compatible with being created at the very center of the galaxy where most of the $\mathrm{H} \alpha$ emission is found. Also, AGN-driven outflows are expected to display higher velocities than the ones observed here (Rupke \& Veilleux 2013; Rupke et al. 2017). Although further investigations are needed, it seems plausible to discard the AGN source as the potential mechanism that generates the outflow in this case.

Finally, the analysis of the ionized and cold phases of the ISM shows that they have different locations within the galaxy. The $\mathrm{H} \alpha$ emission is confined to a disk structure supported by rotation, while the $\mathrm{NaI} \mathrm{D}$ is associated with (i) a shell-like structure infalling toward the galaxy center and (ii) a blueshifted $\mathrm{NaI} \mathrm{D}$ absorption in the near side of the galaxy that reflects the presence of a galactic outflow along the NE semiminor axis. The ionized gas in the central region shares the kinematic pattern of the thin-disk model proposed to explain the motion of the gas. Thus, the analysis of the ionized $\mathrm{H} \alpha$ emission line shows the presence of three different dynamical components, with one of them associated with a highly inclined $\left(i=75^{\circ}\right)$ rotating disk. Also, a second dynamical component associated with the position of the dust lanes is present. The gas is rotating in this dusty structure surrounding the central regions with a higher inclination, $i=83^{\circ}$.

\section{Summary and Conclusions}

In this paper, we presented high-resolution optical IFS observations of the nearby galaxy UGC 10205, made with MEGARA on the GTC $10.4 \mathrm{~m}$ telescope at La Palma Observatory. The combination of the $\mathrm{LR}-V$ and $\mathrm{HR}-R$ setups provided a wealth of information on the state of the multiphase ISM and the stellar kinematics in UGC 10205 and allowed us to study the kinematics of the cool and ionized gas in detail. MEGARA IFS observations have shown an intriguing and complex structure in the central regions of UGC 10205. The main results obtained in this study can be summarized as follows:

1. We present the fundamental parameters of the galaxy kinematics by means of creating 2D stellar kinematics maps for the first two order moments of the line-of-sight velocity distribution ( $V$ and $\sigma$ ).

2. In an attempt to reconciliate the ionized and stellar velocity rotation curves, ADC corrections are applied. Having highquality IFS data is fundamental to properly estimating the ADC in each Voronoi cell and recovering the shape of the SVE. A mean value of $V_{\text {rot }}$ (stars) $/ V_{\text {rot }}$ (gas) $=0.75 \pm 0.08$ was found. Applying this correction, there is a significant agreement between the derived stellar velocity rotation curve and the gaseous ionized $\mathrm{H} \alpha$ one previously derived in the literature by both Vega Beltran et al. (1997) using long-slit spectroscopy data and García-Lorenzo et al. (2015) with the CALIFA data. These results suggest that the innermost regions of early-type spirals needed large values of the ADC, as proposed by Cortese et al. (2014).

3. We also explore the presence of interstellar $\mathrm{Na} \mathrm{I} \lambda \lambda 5890$, 5896 doublet absorption that is commonly used to trace the properties of neutral gas inflows/outflows. The 
complexity of the neutral gas kinematics is revealed by the presence of multiple narrow components. An overall shift of $\Delta V=(114 \pm 5) \mathrm{km} \mathrm{s}^{-1}$ for the foreground redshifted component of the neutral gas is detected through the Na I D absorption. This suggests the presence of mild inflows toward the inner regions. The inflowing gas detection might be triggered by a past minor merger suffered by the galaxy (Reshetnikov \& Evstigneeva 1999).

4. The characterization of outflows is essential to place constraints on the models of galaxy formation and evolution. The majority of works in the literature has focused on understanding the complexity of outflows in extreme objects such as ULIRGS. Here, we point out the necessity to extend these studies to include less active galaxies. To elucidate the role that this outflow might play in removing gas from UGC 10205, we calculate physical properties of the cold gas entrained in the wind applying the formalism of the thin-shell model. We obtained a total mass in the wind $M_{\text {out }}=4.55 \pm 0.06 \times 10^{7} M_{\odot}$ and a cold-gas mass outflow rate $\dot{M}_{\text {out }}=0.78 \pm 0.03 M_{\odot} \mathrm{yr}^{-1}$. The calculation of the mass loading factor yield values of $0.59<\eta<1.59$; although, more accuracy SFR measurements are needed to constraint this value as well as largescale observations to explore the effect of the wind in the neighboring ISM and circumgalactic medium.

5. Finally, assuming that the gas describes circular orbits in the galaxy's plane, we model the $\mathrm{H} \alpha$ gas distribution in the inner regions using a thin-disk model with $\left(i=75^{\circ}\right)$. The primary goal of our modeling is to characterize the kinematics and the ionized gas geometry. Despite the complexity of the $\mathrm{H} \alpha$ emission-line profile where up to three kinematically distinct gaseous components appeared, our simple thin-disk model still can reproduce most of the kinematical features seen in the central regions of UGC 10205. Also, a distinct kinematic component of the gas with a higher inclination $\left(i=83^{\circ}\right)$ pattern is detected in a position coincident with the dust lane structures.

Finally, we emphasize the potential of MEGARA to make meaningful comparisons between the neutral and the ionized gas phase components in the central regions of nearby galaxies.

UGC 10205 is a remarkable example of an object that has revealed an active current phase, reinforcing the importance of spatially resolved high-resolution analyses in less active galaxies. A systematic exploration of Galactic winds in normal star-forming objects and in its multiple phases is required to provide a complete picture on the role that these phenomena might play to shape galaxies and their influence on surrounding environments.

The authors acknowledge financial support from the Spanish Ministry of Economy and Competitiveness (MINECO) under grant No. AYA2016-75808-R, which is partly funded by the European Regional Development Fund (ERDF), AYA201790589-REDT, RTI2018-096188-B-I00, S2018/NMT-4291, and ESP2017-83197P projects. C.C.T. gratefully acknowledges the support of the European Youth Employment Initiative (YEI) by means of the Postdoctoral Fellowship Program. G.B. acknowledges financial support from DGAPAUNAM through PAPIIT project IG100319.

\section{ORCID iDs}

C. Catalán-Torrecilla @ https://orcid.org/0000-00028067-0164

Á. Castillo-Morales (ํ) https://orcid.org/0000-0003-4964-3245

A. Gil de Paz (ㄱ) https://orcid.org/0000-0001-6150-2854

J. Gallego (i) https://orcid.org/0000-0003-1439-7697

J. Iglesias-Páramo (iD https://orcid.org/0000-0003-2726-6370

S. Pascual (1) https://orcid.org/0000-0002-9351-6051

N. Cardiel ๑i https://orcid.org/0000-0002-9334-2979

B. T. Dullo (i) https://orcid.org/0000-0002-4140-0110

P. Coelho (i) https://orcid.org/0000-0003-1846-4826

G. Bruzual (1) https://orcid.org/0000-0002-6971-5755

S. Charlot 1 ib https://orcid.org/0000-0003-3458-2275

\section{References}

Arribas, S., Colina, L., Bellocchi, E., Maiolino, R., \& Villar-Martín, M. 2014, A\&A, 568, A14

Baldwin, J. A., Phillips, M. M., \& Terlevich, R. 1981, PASP, 93, 5

Barai, P., Monaco, P., Murante, G., Ragagnin, A., \& Viel, M. 2015, MNRAS, 447, 266

Barrera-Ballesteros, J. K., García-Lorenzo, B., Falcón-Barroso, J., et al. 2015, A\&A, 582, A21

Binney, J., \& Tremaine, S. 1987, Galactic Dynamics (Princeton, NJ: Princeton Univ. Press)

Blanc, G. A., Weinzirl, T., Song, M., et al. 2013, AJ, 145, 138

Bohlin, R. C., Savage, B. D., \& Drake, J. F. 1978, ApJ, 224, 132

Bruzual, G., \& Charlot, S. 2003, MNRAS, 344, 1000

Calzetti, D., Kennicutt, R. C., Engelbracht, C. W., et al. 2007, ApJ, 666, 870

Cappellari, M. 2017, MNRAS, 466, 798

Cappellari, M., \& Copin, Y. 2003, MNRAS, 342, 345

Cappellari, M., \& Emsellem, E. 2004, PASP, 116, 138

Cardiel, N., \& Pascual, S. 2018, guaix-ucm/megaradrp-calibrations: Release 2018.1, Zenodo, doi:10.5281/zenodo.2270518

Carrasco, E., Gil de Paz, A., Gallego, J., et al. 2018, Proc. SPIE, 10702, 1070216

Castillo-Morales, A., Jiménez-Vicente, J., Mediavilla, E., \& Battaner, E. 2007, MNRAS, 380, 489

Catalán-Torrecilla, C., Gil de Paz, A., Castillo-Morales, A., et al. 2015, A\&A, 584, A87

Catalán-Torrecilla, C., Gil de Paz, A., Castillo-Morales, A., et al. 2017, ApJ, 848,87

Cazzoli, S., Arribas, S., Colina, L., et al. 2014, A\&A, 569, A14

Cazzoli, S., Arribas, S., Maiolino, R., \& Colina, L. 2016, A\&A, 590, A125

Chabrier, G. 2003, PASP, 115, 763

Chambers, K. C., Magnier, E. A., Metcalfe, N., et al. 2016, arXiv:1612.05560

Chen, Y.-M., Tremonti, C. A., Heckman, T. M., et al. 2010, AJ, 140, 445

Ciardullo, R., Durrell, P. R., Laychak, M. B., et al. 2004, ApJ, 614, 167

Coelho, P., Barbuy, B., Meléndez, J., Schiavon, R. P., \& Castilho, B. V. 2005, A\&A, 443, 735

Coelho, P., Bruzual, G., Charlot, S., et al. 2007, MNRAS, 382, 498

Coelho, P. R. T. 2014, MNRAS, 440, 1027

Cortese, L., Fogarty, L. M. R., Ho, I.-T., et al. 2014, ApJL, 795, L37

Courteau, S. 1997, AJ, 114, 2402

Dalcanton, J. J. 2007, ApJ, 658, 941

Dullo, B. T., Chamorro-Cazorla, M., Gil de Paz, A., et al. 2019, ApJ, 871, 9

Falcón-Barroso, J., Lyubenova, M., van de Ven, G., et al. 2017, A\&A, 597, A48

Finkelman, I., Brosch, N., Funes, J. G., et al. 2012, MNRAS, 422, 1384

Finkelman, I., Brosch, N., Funes, J. G., Kniazev, A. Y., \& Väisänen, P. 2010, MNRAS, 407, 2475

García-Lorenzo, B., Márquez, I., Barrera-Ballesteros, J. K., et al. 2015, A\&A, 573, A59

Gentile, G., Tydtgat, C., Baes, M., et al. 2015, A\&A, 576, A57

Gerssen, J., Kuijken, K., \& Merrifield, M. R. 1997, MNRAS, 288, 618

Gerssen, J., Kuijken, K., \& Merrifield, M. R. 2000, MNRAS, 317, 545

Gerssen, J., \& Shapiro Griffin, K. 2012, MNRAS, 423, 2726

Gordon, K. D., Clayton, G. C., Witt, A. N., \& Misselt, K. A. 2000, ApJ, 533,236

Granato, G. L., De Zotti, G., Silva, L., Bressan, A., \& Danese, L. 2004, ApJ, 600,580

Hao, C.-N., Kennicutt, R. C., Johnson, B. D., et al. 2011, ApJ, 741, 124 
Heckman, T. M., Alexandroff, R. M., Borthakur, S., Overzier, R., \& Leitherer, C. 2015, ApJ, 809, 147

Heckman, T. M., Lehnert, M. D., Strickland, D. K., \& Armus, L. 2000, ApJS, 129,493

Herrmann, K. A., \& Ciardullo, R. 2009, ApJ, 705, 1686

Iglesias-Páramo, J., Buat, V., Takeuchi, T. T., et al. 2006, ApJS, 164, 38

Inoue, A. K., Hirashita, H., \& Kamaya, H. 2001, ApJ, 555, 613

Jiménez-Vicente, J., Castillo-Morales, A., Mediavilla, E., \& Battaner, E. 2007, MNRAS, 382, L16

Kalinova, V., Colombo, D., Rosolowsky, E., et al. 2017, MNRAS, 469, 2539

Kauffmann, G., Heckman, T. M., Tremonti, C., et al. 2003, MNRAS, 346, 1055

Kelz, A., Verheijen, M. A. W., Roth, M. M., et al. 2006, PASP, 118, 129

Kennicutt, R. C., \& Evans, N. J. 2012, ARA\&A, 50, 531

Kennicutt, R. C., Hao, C.-N., Calzetti, D., et al. 2009, ApJ, 703, 1672

Kewley, L. J., Dopita, M. A., Sutherland, R. S., Heisler, C. A., \& Trevena, J. 2001, ApJ, 556, 121

King, A., \& Pounds, K. 2015, ARA\&A, 53, 115

Martin, C. L. 2005, ApJ, 621, 227

Martin, C. L. 2006, ApJ, 647, 222

Martín-Fernández, P., Jiménez-Vicente, J., Zurita, A., Mediavilla, E., \& Castillo-Morales, Á 2016, MNRAS, 461, 6

Martinsson, T. P. K., Verheijen, M. A. W., Westfall, K. B., et al. 2013, A\&A, 557, A130

Merrett, H. R., Merrifield, M. R., Douglas, N. G., et al. 2006, MNRAS, 369,120

Mogotsi, K. M., \& Romeo, A. B. 2019, MNRAS, 489, 3797

Muratov, A. L., Kereš, D., Faucher-Giguère, C.-A., et al. 2015, MNRAS, 454, 2691

Nair, P. B., \& Abraham, R. G. 2010, ApJS, 186, 427

Nath, B. B., \& Trentham, N. 1997, MNRAS, 291, 505

Nayakshin, S., \& Zubovas, K. 2012, MNRAS, 427, 372

Nilson, P. 1973, Uppsala General Catalogue of Galaxies (Uppsala: Astronomiska Observatorium)

Noordermeer, E. 2008, MNRAS, 385, 1359

Noordermeer, E., Merrifield, M. R., Coccato, L., et al. 2008, MNRAS, 384, 943

Oppenheimer, B. D., \& Davé, R. 2006, MNRAS, 373, 1265

Oppenheimer, B. D., \& Davé, R. 2008, MNRAS, 387, 577

Pascual, S., Cardiel, N., Picazo-Sanchez, P., Castillo-Morales, A., \& de Paz, A. G. 2018, guaix-ucm/megaradrp: v0.8, Zenodo, doi:10.5281/ zenodo. 2206856

Perna, M., Cresci, G., Brusa, M., et al. 2019, A\&A, 623, A171

Reshetnikov, V. P., \& Evstigneeva, E. A. 1999, ARep, 43, 367

Roberts-Borsani, G. W., \& Saintonge, A. 2019, MNRAS, 482, 4111

Roth, M. M., Kelz, A., Fechner, T., et al. 2005, PASP, 117, 620
Rubin, V. C., Burstein, D., Ford, W. K., Jr., \& Thonnard, N. 1985, ApJ, 289,81

Rubin, V. C. 1987, in IAU Symp. 117, Dark Matter in the Universe, ed J. Kormendy \& G. R. Knapp (Dordrecht: D. Reidel), 51

Rupke, D. 2018, Galax, 6, 138

Rupke, D. S., \& Veilleux, S. 2005, ApJL, 631, L37

Rupke, D. S., Veilleux, S., \& Sanders, D. B. 2002, ApJ, 570, 588

Rupke, D. S., Veilleux, S., \& Sanders, D. B. 2005, ApJS, 160, 115

Rupke, D. S. N., Gültekin, K., \& Veilleux, S. 2017, ApJ, 850, 40

Rupke, D. S. N., \& Veilleux, S. 2013, ApJ, 768, 75

Sánchez, S. F., Kennicutt, R. C., Gil de Paz, A., et al. 2012, A\&A, 538, A8

Sarzi, M., Kaviraj, S., Nedelchev, B., et al. 2016, MNRAS, 456, L25

Schwartz, C. M., \& Martin, C. L. 2004, ApJ, 610, 201

Sérsic, J. L. 1968, Atlas de Galaxias Australes (Córdoba: Observatorio Astronómico)

Shapiro, K. L., Gerssen, J., \& van der Marel, R. P. 2003, AJ, 126, 2707

Shapiro, P. R., \& Field, G. B. 1976, ApJ, 205, 762

Shih, H.-Y., \& Rupke, D. S. N. 2010, ApJ, 724, 1430

Sorce, J. G., Tully, R. B., Courtois, H. M., et al. 2014, MNRAS, 444, 527

Soto, K. T., Martin, C. L., Prescott, M. K. M., \& Armus, L. 2012, ApJ, 757, 86

Strömberg, G. 1924, ApJ, 59, 228

Strömberg, G. 1925, ApJ, 61, 363

Sugahara, Y., Ouchi, M., Lin, L., et al. 2017, ApJ, 850, 51

Tarenghi, M., Garilli, B., \& Maccagni, D. 1994, AJ, 107, 1629

Theureau, G., Bottinelli, L., Coudreau-Durand, N., et al. 1998, A\&As, 130, 333

Turatto, M., Benetti, S., \& Cappellaro, E. 2003, in From Twilight to Highlight: The Physics of Supernovae, ed. W. Hillebrandt \& B. Leibundgut (Berlin: Springer), 200

van Driel, W., Arnaboldi, M., Combes, F., \& Sparke, L. S. 2000, A\&As, 141,385

Vega Beltran, J. C., Corsini, E. M., Pizzella, A., \& Bertola, F. 1997, A\&A, 324,485

Veilleux, S., Cecil, G., \& Bland-Hawthorn, J. 2005, ARA\&A, 43, 769

Vlahakis, C., Dunne, L., \& Eales, S. 2005, MNRAS, 364, 1253

Walcher, C. J., Coelho, P., Gallazzi, A., \& Charlot, S. 2009, MNRAS, 398, L44

Walcher, C. J., Wisotzki, L., Bekeraité, S., et al. 2014, A\&A, 569, A1

Westfall, K. B., Bershady, M. A., Verheijen, M. A. W., et al. 2011, ApJ, 742,18

Westmoquette, M. S., Clements, D. L., Bendo, G. J., \& Khan, S. A. 2012, MNRAS, 424, 416

Whitmore, B. C., Lucas, R. A., McElroy, D. B., et al. 1990, AJ, 100, 1489

Willmer, C. N. A., Rieke, G. H., Le Floc'h, E., et al. 2009, AJ, 138, 146

Wu, C. F. J. 1986, AnSta, 14, 1261

Zubovas, K., Nayakshin, S., King, A., \& Wilkinson, M. 2013, MNRAS, 433, 3079 